\title{
Regioselectivity for Condensation Reactions of Quinonoid Models of Tryptophan Tryptophylquinone: A Density Functional Theory Study
}

\author{
Jian-Wei Zou, Ji-Ming Liang, and Chin-Hui Yu* \\ Department of Chemistry, National Tsing Hua University, \\ Hsinchu 300, Taiwan \\ *e-mail: chyu@oxygen.chem.nthu.edu.tw
}

\section{Table of contents}

Table S1 The optimized geometries of OBQ, MIQ, IIQ at the B3LYP/D95(d,p) level.

Table S2 Some structural parameters of IIQ scanned PES along the dihedral angle $\mathrm{C}_{5} \mathrm{C}_{4} \mathrm{C}_{2}{ }^{\prime} \mathrm{C}_{3}, \chi$, , at the B3LYP/D95(d,p) level.

Table S3 The calculated vibrational frequencies of OBQ, MIQ, and IIQ at the B3LYP/D95(d,p) level scaled by 0.96 .

Appendix 1 Optimized Cartesian coordinates and energies of OBQ, MIQ, IIQ, IIQ', IIQ-TS, the carbinolamine intermediates 6MIQ-I, 7MIQ-I, 6IIQ-I, 7IIQ-I, and 1-16 and their corresponding transition states 6MIQ-TS, 7MIQ-TS, 6IIQ-TS, 7IIQ-TS, and 1TS-16TS.

Appendix 2 Natural population analysis at the B3LYP/D95(d,p) level for OBQ, MIQ and IIQ. 
Table S1 The optimized geometries of OBQ, MIQ, IIQ at the B3LYP/D95(d,p) level (Bond distances in $\AA$, angles in Deg., and $\mathrm{E}_{\text {total }}$ in a.u.).

\begin{tabular}{|c|c|c|c|c|}
\hline \multirow{2}{*}{ Parameter } & \multirow{2}{*}{$\begin{array}{c}\text { OBQ } \\
\text { B3LYP }\end{array}$} & \multirow{2}{*}{$\begin{array}{c}\text { MIQ } \\
\text { B3LYP }\end{array}$} & \multicolumn{2}{|c|}{ IIQ } \\
\hline & & & B3LYP & Exp. $^{a}$ \\
\hline $\mathrm{N}_{1} \mathrm{C}_{2}$ & & 1.367 & 1.366 & 1.370 \\
\hline $\mathrm{N}_{1}{ }^{\prime} \mathrm{C}_{2}{ }^{\prime}$ & & & 1.397 & 1.371 \\
\hline $\mathrm{N}_{1} \mathrm{C}_{8}$ & & 1.371 & 1.370 & 1.377 \\
\hline $\mathrm{N}_{1}{ }^{\prime} \mathrm{C}_{8}{ }^{\prime}$ & & & 1.380 & 1.377 \\
\hline $\mathrm{C}_{2} \mathrm{C}_{3}$ & & 1.400 & 1.400 & 1.362 \\
\hline $\mathrm{C}_{4} \mathrm{C}_{2}$ & & & 1.468 & 1.498 \\
\hline $\mathrm{C}_{4} \mathrm{C}_{5}$ & 1.356 & 1.364 & 1.370 & 1.388 \\
\hline $\mathrm{C}_{6} \mathrm{O}_{1}$ & 1.221 & 1.224 & 1.225 & 1.241 \\
\hline \multicolumn{5}{|l|}{$\mathrm{C}_{6} \mathrm{~N}_{2}$} \\
\hline $\mathrm{C}_{7} \mathrm{O}_{2}$ & 1.221 & 1.227 & 1.227 & 1.233 \\
\hline \multicolumn{5}{|l|}{$\mathrm{C}_{7} \mathrm{~N}_{2}$} \\
\hline $\mathrm{C}_{4} \mathrm{C}_{9}$ & 1.468 & 1.471 & 1.475 & 1.422 \\
\hline $\mathrm{C}_{5} \mathrm{C}_{6}$ & 1.480 & 1.476 & 1.473 & 1.402 \\
\hline $\mathrm{C}_{6} \mathrm{C}_{7}$ & 1.566 & 1.563 & 1.560 & 1.380 \\
\hline $\mathrm{C}_{7} \mathrm{C}_{8}$ & 1.480 & 1.450 & 1.451 & 1.390 \\
\hline $\mathrm{C}_{8} \mathrm{C}_{9}$ & 1.356 & 1.400 & 1.403 & 1.416 \\
\hline \multicolumn{5}{|l|}{$\mathrm{O}_{11} \mathrm{H}$} \\
\hline \multicolumn{5}{|l|}{$\mathrm{O}_{10} \mathrm{H}$} \\
\hline$\angle \mathrm{C}_{2} \mathrm{~N}_{1} \mathrm{C}_{8}$ & & 109.1 & 109.1 & 108.1 \\
\hline$\angle \mathrm{C}_{9} \mathrm{C}_{8} \mathrm{~N}_{1}$ & & 108.5 & 108.5 & 109.1 \\
\hline$\angle \mathrm{C}_{3}{ }^{\prime} \mathrm{C}_{2}{ }^{\prime} \mathrm{N}_{1}{ }^{\prime}$ & & & 109.2 & 111.7 \\
\hline$\angle \mathrm{C}_{5} \mathrm{C}_{6} \mathrm{C}_{7}$ & 117.2 & 118.0 & 117.9 & 120.0 \\
\hline$\angle \mathrm{C}_{6} \mathrm{C}_{7} \mathrm{C}_{8}$ & 117.2 & 113.4 & 113.5 & 118.8 \\
\hline$\angle \mathrm{C}_{7} \mathrm{C}_{8} \mathrm{C}_{9}$ & 120.6 & 125.9 & 126.2 & 122.5 \\
\hline$\angle \mathrm{C}_{5} \mathrm{C}_{4} \mathrm{C}_{9}$ & 122.3 & 119.2 & 119.3 & 119.6 \\
\hline$\angle \mathrm{O}_{10} \mathrm{C}_{6} \mathrm{C}_{7}$ & 120.1 & 119.3 & 119.5 & 119.6 \\
\hline$\angle \mathrm{O}_{11} \mathrm{C}_{7} \mathrm{C}_{6}$ & 120.1 & 122.2 & 122.3 & 122.3 \\
\hline \multicolumn{5}{|l|}{$\angle \mathrm{C}_{6} \mathrm{~N}_{10} \mathrm{H}$} \\
\hline \multicolumn{5}{|l|}{$\angle \mathrm{C}_{7} \mathrm{~N}_{11} \mathrm{H}$} \\
\hline$\chi^{b}$ & & & 49.6 & $51.6^{c}$ \\
\hline $\mathrm{E}_{\text {total }}$ & -381.506571 & -591.767942 & & \\
\hline
\end{tabular}

${ }^{a}$ No experimental structure of IIQ is available. The data is the X-ray crystal structure of TTQ in MADH from Ref.10. ${ }^{b} \chi$ is a dihedral angle $\left(\angle \mathrm{C} 5 \mathrm{C} 4 \mathrm{C} 2{ }^{\prime} \mathrm{C} 3{ }^{\prime}\right)$ of IIQ. ${ }^{c}$ There are two TTQ cofactors per protein and another one with the dihedral angle $57.3^{\circ}$. 
Table S2 Some structural parameters of IIQ scanned PES along the dihedral angle $\mathrm{C}_{5} \mathrm{C}_{4} \mathrm{C}_{2}{ }^{\prime} \mathrm{C}_{3}$,,$\chi$, at the B3LYP/D95(d,p) level (Bond distances in $\AA$, angles in Deg., and $\Delta \mathrm{E}$ in kcal/mo)

\begin{tabular}{|c|c|c|c|c|c|c|c|c|c|c|c|c|}
\hline$\chi$ & $\mathrm{C}_{6} \mathrm{O}_{10}$ & $\mathrm{C}_{7} \mathrm{O}_{11}$ & $\mathrm{C}_{4} \mathrm{C}_{5}$ & $\mathrm{C}_{4} \mathrm{C}_{9}$ & $\mathrm{C}_{5} \mathrm{C}_{6}$ & $\mathrm{C}_{6} \mathrm{C}_{7}$ & $\mathrm{C}_{4} \mathrm{C}_{2}{ }^{\prime}$ & $\angle \mathrm{C}_{5} \mathrm{C}_{4} \mathrm{C}_{2}$ & $\angle \mathrm{C}_{4} \mathrm{C}_{2}{ }^{\prime} \mathrm{C}_{3}{ }^{\prime}$ & $\angle \mathrm{C}_{9} \mathrm{C}_{4} \mathrm{C}_{2}$ & $\angle \mathrm{C}_{4} \mathrm{C}_{2}{ }^{\prime} \mathrm{N}_{1}$ & $\Delta \mathrm{E}$ \\
\hline 0.0 & 1.228 & 1.227 & 1.378 & 1.492 & 1.465 & 1.551 & 1.459 & 120.6 & 131.4 & 121.8 & 119.2 & 5.30 \\
\hline 5.0 & 1.228 & 1.227 & 1.377 & 1.490 & 1.466 & 1.552 & 1.460 & 120.5 & 131.4 & 121.6 & 119.1 & 4.35 \\
\hline 10.0 & 1.228 & 1.227 & 1.377 & 1.488 & 1.466 & 1.553 & 1.460 & 120.4 & 131.4 & 121.5 & 119.2 & 3.45 \\
\hline $30.0^{a}$ & 1.227 & 1.227 & 1.374 & 1.481 & 1.469 & 1.556 & 1.462 & 119.7 & 130.8 & 121.4 & 120.0 & 0.81 \\
\hline 49.6 & 1.225 & 1.227 & 1.370 & 1.475 & 1.473 & 1.560 & 1.468 & 119.4 & 130.4 & 121.2 & 120.4 & 0.00 \\
\hline 70.0 & 1.224 & 1.228 & 1.366 & 1.472 & 1.478 & 1.563 & 1.477 & 119.9 & 130.3 & 120.5 & 120.1 & 0.56 \\
\hline 80.0 & 1.223 & 1.227 & 1.365 & 1.470 & 1.480 & 1.564 & 1.482 & 120.1 & 130.2 & 120.2 & 119.9 & 1.07 \\
\hline 90.0 & 1.223 & 1.227 & 1.364 & 1.469 & 1.482 & 1.565 & 1.485 & 120.3 & 130.3 & 120.1 & 119.7 & 1.52 \\
\hline 100.0 & 1.223 & 1.227 & 1.364 & 1.468 & 1.481 & 1.565 & 1.485 & 119.8 & 130.4 & 120.6 & 119.9 & 1.70 \\
\hline 120.0 & 1.225 & 1.227 & 1.371 & 1.472 & 1.474 & 1.560 & 1.474 & 118.8 & 130.9 & 121.8 & 119.4 & 0.78 \\
\hline 123.5 & 1.226 & 1.227 & 1.372 & 1.473 & 1.473 & 1.560 & 1.472 & 118.7 & 131.1 & 122.0 & 119.3 & 0.74 \\
\hline 130.0 & 1.226 & 1.228 & 1.373 & 1.474 & 1.472 & 1.558 & 1.470 & 118.6 & 131.4 & 122.3 & 119.3 & 0.88 \\
\hline 140.0 & 1.227 & 1.228 & 1.375 & 1.477 & 1.470 & 1.556 & 1.467 & 118.3 & 132.1 & 122.8 & 119.0 & 1.60 \\
\hline $150.0^{b}$ & 1.227 & 1.228 & 1.377 & 1.480 & 1.468 & 1.555 & 1.465 & 118.2 & 132.7 & 123.4 & 118.7 & 2.99 \\
\hline $180.0^{c}$ & 1.227 & 1.227 & 1.380 & 1.489 & 1.468 & 1.554 & 1.464 & 119.2 & 133.8 & 124.3 & 117.5 & 11.32 \\
\hline
\end{tabular}

${ }^{a}$ There is that threshold has not yet satisfy default, i.e., maximum displacement $=0.002426(\AA)$.

${ }^{b}$ There is that threshold has not yet satisfy default, i.e., maximum force $=0.002426$ (Hartree/Bohr of radian).

${ }^{c}$ There is that threshold has not yet satisfy default, i.e., maximum displacement $=0.002426(\AA)$. 
Table S3 The calculated vibrational frequencies (in $\mathrm{cm}^{-1}$ ) of the OBQ, MIQ, and IIQ at the B3LYP/D95(d,p) level scaled by 0.96 (predicted infrared intersities, in mDyne/A, are listed in parentheses).

\begin{tabular}{|c|c|c|c|c|c|c|c|}
\hline \multicolumn{3}{|c|}{ OBQ } & \multicolumn{3}{|c|}{ MIQ } & \multicolumn{2}{|c|}{ IIQ } \\
\hline normal mode & sym. & $\mathrm{v}$ & normal mode & sym. & $v$ & normal mode & $v$ \\
\hline $\mathrm{CO}$ asym-str & $\mathrm{B}_{2}$ & 1703 (65.6) & CO asym-str & $\mathrm{A}^{\prime}$ & $1689(41.0)$ & $\mathrm{CO}$ asym-str & $1683(19.2)$ \\
\hline CO sym-str & $A_{1}$ & 1679 (220.4) & CO sym-str & $A^{\prime}$ & $1665(498.4)$ & CO sym-str & $1658(683.8)$ \\
\hline $\mathrm{C}_{4} \mathrm{C}_{5}, \mathrm{C}_{8} \mathrm{C}_{9}$ asym-str & $\mathrm{B}_{2}$ & $1606(0.2)$ & $\mathrm{C}_{4} \mathrm{C}_{5} \mathrm{str}$ & $\mathrm{A}^{\prime}$ & $1580(65.3)$ & $\mathrm{C}_{4} \mathrm{C}_{5} \mathrm{str}$ & $1556(158.5)$ \\
\hline $\mathrm{C}_{4} \mathrm{C}_{5}, \mathrm{C}_{8} \mathrm{C}_{9}$ sym-str & $\mathrm{A}_{1}$ & $1550(2.2)$ & $\mathrm{C}_{4} \mathrm{C}_{5}, \mathrm{C}_{8} \mathrm{C}_{9}$ sym-str & $A^{\prime}$ & $1521(14.9)$ & $\mathrm{C}_{2}{ }^{\prime} \mathrm{C}_{3}{ }^{\prime} \mathrm{str}$ & $1526(102.1)$ \\
\hline & & & $\mathrm{C}_{2} \mathrm{C}_{3}$ str & $A^{\prime}$ & $1476(37.8)$ & $\mathrm{C}_{4} \mathrm{C}_{5}, \mathrm{C}_{8} \mathrm{C}_{9}$ sym-str & $1508(52.4)$ \\
\hline
\end{tabular}




\section{Appendix 1}

\section{(Optimized Cartesian Coordinates and energies)}

\begin{tabular}{|c|c|c|c|}
\hline OBQ & \multicolumn{2}{|c|}{ (B3LYP/D95(d,p) } & $E=-381.506571$ a.u. \\
\hline $\mathrm{C}$ & 0.783111 & 0.000000 & 0.677260 \\
\hline $\mathrm{C}$ & 1.458395 & 0.000000 & -0.639377 \\
\hline $\mathrm{C}$ & 0.734177 & 0.000000 & -1.785809 \\
\hline $\mathrm{C}$ & -0.734177 & 0.000000 & -1.785809 \\
\hline $\mathrm{C}$ & -1.458395 & 0.000000 & -0.639377 \\
\hline $\mathrm{C}$ & -0.783111 & 0.000000 & 0.677260 \\
\hline $\mathrm{O}$ & 1.395093 & 0.000000 & 1.734282 \\
\hline $\mathrm{H}$ & 2.546090 & 0.000000 & -0.636468 \\
\hline $\mathrm{H}$ & 1.239943 & 0.000000 & -2.750237 \\
\hline $\mathrm{H}$ & -1.239943 & 0.000000 & -2.750237 \\
\hline $\mathrm{H}$ & -2.546090 & 0.000000 & -0.636468 \\
\hline $\mathrm{O}$ & -1.395093 & 0.000000 & 1.734282 \\
\hline MIQ & \multicolumn{2}{|c|}{ (B3LYP/D95(d,p) } & $=-591.767942$ a.u. \\
\hline $\mathrm{C}$ & 0.000000 & 0.000000 & 0.000000 \\
\hline $\mathrm{C}$ & 0.000000 & 0.000000 & 1.476230 \\
\hline $\mathrm{C}$ & 1.381015 & 0.000000 & -0.733182 \\
\hline $\mathrm{O}$ & -1.030178 & -0.000000 & -0.661478 \\
\hline $\mathrm{C}$ & 1.131966 & 0.000000 & 2.236958 \\
\hline $\mathrm{C}$ & 2.513269 & 0.000000 & 0.172524 \\
\hline $\mathrm{H}$ & -0.984128 & -0.000000 & 1.938995 \\
\hline $\mathrm{O}$ & 1.471111 & -0.000000 & -1.957048 \\
\hline $\mathrm{C}$ & 2.444628 & 0.000000 & 1.572207 \\
\hline $\mathrm{N}$ & 3.833377 & -0.000000 & -0.198730 \\
\hline $\mathrm{C}$ & 1.047906 & 0.000000 & 3.741328 \\
\hline $\mathrm{C}$ & 3.793072 & -0.000000 & 2.057394 \\
\hline $\mathrm{C}$ & 4.613054 & 0.000000 & 0.924076 \\
\hline $\mathrm{H}$ & 4.142425 & -0.000000 & -1.162702 \\
\hline $\mathrm{H}$ & 1.546598 & 0.881779 & 4.162132 \\
\hline $\mathrm{H}$ & 1.546598 & -0.881779 & 4.162132 \\
\hline $\mathrm{H}$ & 0.007192 & 0.000000 & 4.075673 \\
\hline $\mathrm{H}$ & 5.693643 & -0.000000 & 0.863872 \\
\hline
\end{tabular}




$\begin{array}{lrrr}\mathrm{C} & 4.314499 & -0.000000 & 3.470071 \\ \mathrm{H} & 5.409306 & -0.000000 & 3.472147 \\ \mathrm{H} & 3.983164 & -0.883916 & 4.027348 \\ \mathrm{H} & 3.983164 & 0.883916 & 4.027348\end{array}$

\begin{tabular}{|c|c|c|c|}
\hline IIQ & \multicolumn{2}{|c|}{ (B3LYP/D95 $(d, p)$} & $E=-954.438925$ a.u. \\
\hline $\mathrm{C}$ & 0.000000 & 0.000000 & 0.000000 \\
\hline $\mathrm{C}$ & 0.000000 & 0.000000 & 1.473080 \\
\hline $\mathrm{C}$ & 1.379155 & 0.000000 & -0.728671 \\
\hline $\mathrm{O}$ & -1.032084 & 0.032256 & -0.660060 \\
\hline $\mathrm{C}$ & 1.135928 & -0.010105 & 2.239434 \\
\hline $\mathrm{C}$ & 2.511914 & -0.040365 & 0.176931 \\
\hline $\mathrm{H}$ & -0.977619 & 0.086689 & 1.938151 \\
\hline $\mathrm{O}$ & 1.474402 & 0.001207 & -1.952358 \\
\hline $\mathrm{C}$ & 2.452630 & -0.084417 & 1.577952 \\
\hline $\mathrm{C}$ & 1.021556 & 0.080368 & 3.700221 \\
\hline $\mathrm{N}$ & 3.820083 & -0.179194 & -0.207737 \\
\hline $\mathrm{C}$ & 3.793812 & -0.288395 & 2.047075 \\
\hline $\mathrm{C}$ & 4.599430 & -0.331600 & 0.903334 \\
\hline $\mathrm{H}$ & 4.115358 & -0.201229 & -1.175958 \\
\hline $\mathrm{C}$ & 0.229740 & -0.639011 & 4.588395 \\
\hline $\mathrm{N}$ & 1.764469 & 1.026963 & 4.409931 \\
\hline $\mathrm{C}$ & 4.324144 & -0.495591 & 3.441891 \\
\hline $\mathrm{H}$ & 5.669627 & -0.475442 & 0.829883 \\
\hline $\mathrm{C}$ & -0.684512 & -1.792768 & 4.288618 \\
\hline $\mathrm{H}$ & 2.292913 & 1.769565 & 3.975436 \\
\hline $\mathrm{C}$ & 0.495056 & -0.100413 & 5.899419 \\
\hline $\mathrm{C}$ & 1.440261 & 0.955178 & 5.749657 \\
\hline $\mathrm{C}$ & 1.892727 & 1.716330 & 6.838866 \\
\hline $\mathrm{C}$ & 0.011671 & -0.412870 & 7.188464 \\
\hline $\mathrm{H}$ & 4.411953 & 0.442190 & 4.002070 \\
\hline $\mathrm{H}$ & 5.320051 & -0.949170 & 3.400423 \\
\hline $\mathrm{H}$ & 3.674125 & -1.156433 & 4.023606 \\
\hline $\mathrm{H}$ & -0.576153 & -2.134316 & 3.255699 \\
\hline $\mathrm{H}$ & -0.473179 & -2.640342 & 4.952584 \\
\hline $\mathrm{H}$ & -1.736494 & -1.516830 & 4.440221 \\
\hline $\mathrm{H}$ & 2.608469 & 2.525998 & 6.711124 \\
\hline $\mathrm{C}$ & 1.391243 & 1.390865 & 8.101191 \\
\hline
\end{tabular}




$\begin{array}{lrcc}\mathrm{C} & 0.462177 & 0.335042 & 8.275649 \\ \mathrm{H} & -0.707263 & -1.217022 & 7.331084 \\ \mathrm{H} & 1.721994 & 1.958050 & 8.968797 \\ \mathrm{H} & 0.096395 & 0.111550 & 9.275539\end{array}$

\begin{tabular}{|c|c|c|c|}
\hline IIQ' & \multicolumn{2}{|c|}{ B3LYP/D95(d,p) } & 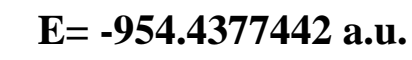 \\
\hline $\mathrm{C}$ & 2.656456 & -2.070055 & 0.242502 \\
\hline $\mathrm{C}$ & 1.221255 & -1.749721 & 0.154767 \\
\hline $\mathrm{C}$ & 3.671922 & -0.889153 & 0.158658 \\
\hline $\mathrm{O}$ & 3.068834 & -3.212816 & 0.403734 \\
\hline $\mathrm{C}$ & 0.726115 & -0.478634 & 0.009676 \\
\hline $\mathrm{C}$ & 3.041396 & 0.400882 & -0.046794 \\
\hline $\mathrm{H}$ & 0.550148 & -2.591560 & 0.304042 \\
\hline $\mathrm{O}$ & 4.885251 & -1.062813 & 0.223222 \\
\hline $\mathrm{C}$ & 1.664374 & 0.642948 & -0.165737 \\
\hline $\mathrm{C}$ & -0.732924 & -0.282749 & 0.011411 \\
\hline $\mathrm{N}$ & 3.711494 & 1.568019 & -0.305129 \\
\hline $\mathrm{C}$ & 1.512273 & 2.017855 & -0.546531 \\
\hline $\mathrm{C}$ & 2.807006 & 2.545022 & -0.610797 \\
\hline $\mathrm{H}$ & 4.721242 & 1.641799 & -0.301158 \\
\hline $\mathrm{C}$ & -1.546909 & 0.512352 & 0.808735 \\
\hline $\mathrm{N}$ & -1.534282 & -1.099587 & -0.789559 \\
\hline $\mathrm{C}$ & 0.276825 & 2.806234 & -0.890665 \\
\hline $\mathrm{H}$ & 3.121366 & 3.548561 & -0.866557 \\
\hline $\mathrm{C}$ & -1.135075 & 1.420716 & 1.932005 \\
\hline $\mathrm{H}$ & -1.174756 & -1.690462 & -1.525745 \\
\hline $\mathrm{C}$ & -2.906554 & 0.191494 & 0.453460 \\
\hline $\mathrm{C}$ & -2.863265 & -0.805034 & -0.564436 \\
\hline $\mathrm{C}$ & -4.026038 & -1.322344 & -1.156856 \\
\hline $\mathrm{C}$ & -4.160108 & 0.656248 & 0.908028 \\
\hline $\mathrm{H}$ & -0.403984 & 2.227955 & -1.522593 \\
\hline $\mathrm{H}$ & 0.551169 & 3.719458 & -1.429440 \\
\hline $\mathrm{H}$ & -0.284364 & 3.102118 & 0.001205 \\
\hline $\mathrm{H}$ & -0.050794 & 1.554641 & 1.972017 \\
\hline $\mathrm{H}$ & -1.601502 & 2.409939 & 1.837946 \\
\hline $\mathrm{H}$ & -1.453220 & 1.007869 & 2.898293 \\
\hline $\mathrm{H}$ & -3.980014 & -2.078061 & -1.938365 \\
\hline $\mathrm{C}$ & -5.251228 & -0.836904 & -0.695381 \\
\hline
\end{tabular}




$\begin{array}{lrrc}\mathrm{C} & -5.318785 & 0.140468 & 0.329138 \\ \mathrm{H} & -4.222349 & 1.406273 & 1.693942 \\ \mathrm{H} & -6.173313 & -1.219820 & -1.127748 \\ \mathrm{H} & -6.293199 & 0.489541 & 0.664221\end{array}$

\begin{tabular}{|c|c|c|c|}
\hline IIQ-TS & \multicolumn{2}{|c|}{ B3LYP/D95(d,p) } & $E=-954.436203$ a.u. \\
\hline $\mathrm{C}$ & 2.842975 & -1.950680 & .001038 \\
\hline $\mathrm{C}$ & 1.373901 & -1.759249 & .041894 \\
\hline $\mathrm{C}$ & 3.751465 & -.679314 & -.090836 \\
\hline $\mathrm{O}$ & 3.358081 & -3.059094 & .038944 \\
\hline $\mathrm{C}$ & .776694 & -.533809 & .001863 \\
\hline $\mathrm{C}$ & 3.002560 & .559696 & -.134167 \\
\hline $\mathrm{O}$ & 4.975556 & -.758998 & -.128201 \\
\hline $\mathrm{C}$ & 1.605381 & .674318 & -.101265 \\
\hline $\mathrm{C}$ & -.704731 & -.427743 & .041544 \\
\hline $\mathrm{N}$ & 3.534412 & 1.819718 & -.240914 \\
\hline $\mathrm{C}$ & 1.289466 & 2.066968 & -.197715 \\
\hline $\mathrm{C}$ & 2.517320 & 2.733139 & -.281404 \\
\hline $\mathrm{C}$ & -1.554923 & -.169686 & 1.098127 \\
\hline $\mathrm{N}$ & -1.444695 & -.579380 & -1.130717 \\
\hline $\mathrm{C}$ & -.054028 & 2.743887 & -.213797 \\
\hline $\mathrm{C}$ & -1.176596 & .064563 & 2.532771 \\
\hline $\mathrm{C}$ & -2.893865 & -.170752 & .553316 \\
\hline $\mathrm{C}$ & -2.789630 & -.434538 & -.842781 \\
\hline $\mathrm{C}$ & -3.914327 & -.511332 & -1.678483 \\
\hline $\mathrm{C}$ & -4.170869 & .023929 & 1.120014 \\
\hline $\mathrm{C}$ & -5.166460 & -.315693 & -1.090287 \\
\hline $\mathrm{C}$ & -5.293903 & -.049423 & .294648 \\
\hline $\mathrm{H}$ & .782465 & -2.667328 & .125746 \\
\hline $\mathrm{H}$ & 4.528466 & 2.005408 & -.287810 \\
\hline $\mathrm{H}$ & 2.713325 & 3.794107 & -.367000 \\
\hline $\mathrm{H}$ & -1.052488 & -.835812 & -2.025014 \\
\hline $\mathrm{H}$ & -.706282 & 2.327214 & -.988767 \\
\hline $\mathrm{H}$ & .060146 & 3.816078 & -.404968 \\
\hline $\mathrm{H}$ & -.576459 & 2.622845 & .741276 \\
\hline $\mathrm{H}$ & -.093954 & -.005778 & 2.676178 \\
\hline $\mathrm{H}$ & -1.498706 & 1.056735 & 2.876942 \\
\hline $\mathrm{H}$ & -1.648755 & -.674435 & 3.192659 \\
\hline
\end{tabular}




$\begin{array}{lrrc}\mathrm{H} & -3.820704 & -.717381 & -2.742989 \\ \mathrm{H} & -4.280440 & .226131 & 2.183701 \\ \mathrm{H} & -6.060471 & -.369232 & -1.708118 \\ \mathrm{H} & -6.285841 & .098131 & .716756\end{array}$

\section{Carbinolamine intermediates}

\begin{tabular}{|c|c|c|c|}
\hline 6MIQ-I & (B3LYP/I & $95++(d, p))$ & $E=-648.36742$ a.u. \\
\hline $\mathrm{C}$ & 0.000000 & 0.000000 & 0.000000 \\
\hline $\mathrm{C}$ & 0.000000 & 0.000000 & 1.514580 \\
\hline $\mathrm{C}$ & 1.403143 & 0.000000 & -0.657737 \\
\hline $\mathrm{O}$ & -0.675643 & 1.199037 & -0.410847 \\
\hline $\mathrm{N}$ & -0.624535 & -1.227427 & -0.469023 \\
\hline $\mathrm{C}$ & 1.096275 & -0.129754 & 2.291366 \\
\hline $\mathrm{C}$ & 2.505539 & -0.172965 & 0.240578 \\
\hline $\mathrm{H}$ & -0.984355 & 0.105359 & 1.968937 \\
\hline $\mathrm{O}$ & 1.492857 & 0.139630 & -1.885173 \\
\hline $\mathrm{H}$ & -0.467646 & 1.319140 & -1.353941 \\
\hline $\mathrm{H}$ & -1.591400 & -1.278388 & -0.153306 \\
\hline $\mathrm{H}$ & -0.602639 & -1.264626 & -1.488371 \\
\hline $\mathrm{C}$ & 2.412122 & -0.240507 & 1.639820 \\
\hline $\mathrm{C}$ & 0.989416 & -0.144945 & 3.797960 \\
\hline $\mathrm{N}$ & 3.831148 & -0.263797 & -0.116372 \\
\hline $\mathrm{C}$ & 3.746503 & -0.386354 & 2.136953 \\
\hline $\mathrm{C}$ & 4.581000 & -0.397742 & 1.015837 \\
\hline $\mathrm{H}$ & 4.160434 & -0.238573 & -1.072504 \\
\hline $\mathrm{H}$ & 1.563257 & 0.674502 & 4.248392 \\
\hline $\mathrm{H}$ & 1.377446 & -1.081582 & 4.217297 \\
\hline $\mathrm{H}$ & -0.053747 & -0.040010 & 4.110970 \\
\hline $\mathrm{C}$ & 4.244295 & -0.510709 & 3.553660 \\
\hline $\mathrm{H}$ & 5.658849 & -0.493325 & 0.970071 \\
\hline $\mathrm{H}$ & 3.989504 & 0.367975 & 4.157952 \\
\hline $\mathrm{H}$ & 5.334923 & -0.612692 & 3.564453 \\
\hline $\mathrm{H}$ & 3.826310 & -1.389311 & 4.059324 \\
\hline 7MIQ-I & \multicolumn{2}{|c|}{$($ B3LYP/D95++(d,p)) } & $E=-648.36542$ a.u. \\
\hline $\mathrm{C}$ & 0.000000 & 0.000000 & 0.000000 \\
\hline
\end{tabular}




$\begin{array}{lrrc}\mathrm{C} & 0.000000 & 0.000000 & 1.451840 \\ \mathrm{C} & 1.360616 & 0.000000 & -0.758716 \\ \mathrm{O} & -1.015390 & -0.038742 & -0.703177 \\ \mathrm{C} & 1.126964 & 0.238827 & 2.197838 \\ \mathrm{C} & 2.471726 & 0.430504 & 0.146542 \\ \mathrm{H} & -0.966020 & -0.141742 & 1.930515 \\ \mathrm{~N} & 1.549525 & -1.396907 & -1.131566 \\ \mathrm{O} & 1.291675 & 0.901609 & -1.863307 \\ \mathrm{C} & 2.397930 & 0.502160 & 1.533823 \\ \mathrm{C} & 1.039738 & 0.261473 & 3.701717 \\ \mathrm{~N} & 3.734966 & 0.753890 & -0.242137 \\ \mathrm{H} & 0.760857 & -1.715134 & -1.694722 \\ \mathrm{H} & 2.410130 & -1.521653 & -1.661530 \\ \mathrm{H} & 0.375657 & 0.841822 & -2.192117 \\ \mathrm{C} & 3.710868 & 0.892705 & 2.006897 \\ \mathrm{C} & 4.496278 & 1.045136 & 0.879707 \\ \mathrm{H} & 4.024839 & 0.875832 & -1.203168 \\ \mathrm{H} & 1.334755 & 1.240850 & 4.097829 \\ \mathrm{H} & 1.720578 & -0.478445 & 4.140748 \\ \mathrm{H} & 0.023626 & 0.042622 & 4.040836 \\ \mathrm{C} & 4.194248 & 1.123217 & 3.413873 \\ \mathrm{H} & 5.531609 & 1.346293 & 0.789966 \\ \mathrm{H} & 3.637872 & 1.925991 & 3.913783 \\ \mathrm{H} & 5.251249 & 1.410360 & 3.407838 \\ \mathrm{H} & 4.100131 & 0.223774 & 4.034929\end{array}$

\begin{tabular}{llrc} 
6IIQ-I & \multicolumn{4}{l}{ (B3LYP/D95(d,p) } & $\mathbf{E}=\mathbf{- 1 0 1 1 . 0 2 1 4 5}$ \\
$\mathrm{C}$ & 0.000000 & 0.000000 & 0.000000 \\
$\mathrm{C}$ & 0.000000 & 0.000000 & 1.550000 \\
$\mathrm{C}$ & 1.373526 & 0.000000 & 2.191624 \\
$\mathrm{C}$ & 2.534183 & -0.194603 & 1.524081 \\
$\mathrm{C}$ & 2.492000 & -0.380624 & 0.063483 \\
$\mathrm{C}$ & 1.267743 & -0.260461 & -0.615206 \\
$\mathrm{~N}$ & 1.493739 & -0.453911 & -1.958663 \\
$\mathrm{C}$ & 3.487625 & -0.685061 & -0.916797 \\
$\mathrm{C}$ & 2.820417 & -0.719130 & -2.147184 \\
$\mathrm{C}$ & 3.821977 & -0.188381 & 2.253430 \\
$\mathrm{~N}$ & 4.749311 & 0.831742 & 2.027584
\end{tabular}




$\begin{array}{lrrc}\mathrm{C} & 4.330259 & -1.073234 & 3.186904 \\ \mathrm{C} & 5.632639 & -0.569831 & 3.562816 \\ \mathrm{C} & 5.853971 & 0.635689 & 2.836274 \\ \mathrm{C} & 7.021775 & 1.398742 & 2.985919 \\ \mathrm{C} & 6.625104 & -1.021270 & 4.456860 \\ \mathrm{C} & 7.984670 & 0.933174 & 3.886188 \\ \mathrm{C} & 7.795634 & -0.274330 & 4.600931 \\ \mathrm{~N} & -0.679552 & -1.235733 & 1.916090 \\ \mathrm{O} & -0.656300 & 1.191882 & 1.993225 \\ \mathrm{O} & -1.067646 & 0.188370 & -0.599809 \\ \mathrm{H} & -1.586707 & -1.278984 & 1.451765 \\ \mathrm{H} & -0.827414 & -1.267112 & 2.922805 \\ \mathrm{H} & -1.396576 & 1.329485 & 1.376957 \\ \mathrm{H} & 1.386364 & 0.174785 & 3.265416 \\ \mathrm{H} & 0.766520 & -0.431567 & -2.660641 \\ \mathrm{H} & 3.220851 & -0.923668 & -3.132428 \\ \mathrm{C} & 4.957138 & -0.956634 & -0.738455 \\ \mathrm{C} & 3.694392 & -2.338263 & 3.688280 \\ \mathrm{H} & 6.483324 & -1.938306 & 5.024916 \\ \mathrm{H} & 8.570568 & -0.609875 & 5.286957 \\ \mathrm{H} & 8.902472 & 1.500240 & 4.026955 \\ \mathrm{H} & 7.177654 & 2.317598 & 2.424538 \\ \mathrm{H} & 4.548269 & 1.669437 & 1.500376 \\ \mathrm{H} & 5.514235 & -0.052424 & -0.467784 \\ \mathrm{H} & 5.387414 & -1.353024 & -1.664070 \\ \mathrm{H} & 5.132327 & -1.687103 & 0.058255 \\ \mathrm{H} & 2.733663 & -2.519033 & 3.197203 \\ \mathrm{H} & 4.339486 & -3.207358 & 3.503526 \\ \mathrm{H} & 3.516397 & -2.292807 & 4.770803\end{array}$

\section{IIQ-I}

$\mathrm{C}$

C

C

C

C

C

$\mathrm{N}$
$($ B3LYP/D95(d,p)) $\quad E=-\mathbf{1 0 1 1 . 0 1 7 8 2 ~ a . u . ~}$

$\begin{array}{lll}0.000000 & 0.000000 & 0.000000\end{array}$

$\begin{array}{lll}0.000000 & 0.000000 & 1.552000\end{array}$

$\begin{array}{lll}1.264193 & 0.000000 & 2.264191\end{array}$

$\begin{array}{lll}2.471129 & 0.244299 & 1.643493\end{array}$

$\begin{array}{lll}2.527488 & 0.452711 & 0.197522\end{array}$

$\begin{array}{lll}1.339728 & 0.402473 & -0.533060\end{array}$

$\begin{array}{lll}1.626213 & 0.604000 & -1.847181\end{array}$ 


\begin{tabular}{|c|c|c|c|}
\hline $\mathrm{C}$ & 3.599995 & 0.672509 & -0.757792 \\
\hline $\mathrm{C}$ & 2.993459 & 0.775045 & -1.996782 \\
\hline $\mathrm{C}$ & 3.673295 & 0.354451 & 2.485781 \\
\hline $\mathrm{N}$ & 4.482063 & 1.490476 & 2.421305 \\
\hline $\mathrm{C}$ & 4.182144 & -0.516435 & 3.440743 \\
\hline $\mathrm{C}$ & 5.357195 & 0.118763 & 3.986664 \\
\hline $\mathrm{C}$ & 5.503168 & 1.381779 & 3.343221 \\
\hline $\mathrm{C}$ & 6.539394 & 2.272299 & 3.666374 \\
\hline $\mathrm{C}$ & 6.295208 & -0.263817 & 4.968848 \\
\hline $\mathrm{C}$ & 7.448718 & 1.870535 & 4.647865 \\
\hline $\mathrm{C}$ & 7.331129 & 0.613677 & 5.289623 \\
\hline $\mathrm{N}$ & -0.240853 & -1.398905 & -0.341549 \\
\hline $\mathrm{O}$ & -0.989210 & 0.906317 & -0.480290 \\
\hline $\mathrm{O}$ & -1.109293 & -0.004403 & 2.099374 \\
\hline $\mathrm{H}$ & -1.114471 & -1.707919 & 0.084716 \\
\hline $\mathrm{H}$ & -0.306013 & -1.520359 & -1.350175 \\
\hline $\mathrm{H}$ & -1.709631 & 0.862070 & 0.175195 \\
\hline $\mathrm{H}$ & 1.206836 & -0.066247 & 3.346650 \\
\hline $\mathrm{H}$ & 0.931100 & 0.707197 & -2.574016 \\
\hline $\mathrm{H}$ & 3.434983 & 0.949622 & -2.969047 \\
\hline $\mathrm{C}$ & 5.091903 & 0.729665 & -0.552858 \\
\hline $\mathrm{C}$ & 3.673232 & -1.879585 & 3.813347 \\
\hline $\mathrm{H}$ & 6.206813 & -1.222682 & 5.475314 \\
\hline $\mathrm{H}$ & 8.063043 & 0.334458 & 6.044661 \\
\hline $\mathrm{H}$ & 8.264436 & 2.535685 & 4.923410 \\
\hline $\mathrm{H}$ & 6.640548 & 3.234152 & 3.168026 \\
\hline $\mathrm{H}$ & 4.247820 & 2.320288 & 1.897260 \\
\hline $\mathrm{H}$ & 5.425839 & 1.681623 & -0.124514 \\
\hline $\mathrm{H}$ & 5.603833 & 0.604515 & -1.512697 \\
\hline $\mathrm{H}$ & 5.436683 & -0.062564 & 0.119841 \\
\hline $\mathrm{H}$ & 2.846696 & -2.189205 & 3.168659 \\
\hline $\mathrm{H}$ & 4.469847 & -2.630651 & 3.730240 \\
\hline $\mathrm{H}$ & 3.315639 & -1.899940 & 4.851284 \\
\hline 1 & \multicolumn{2}{|c|}{$($ B3LYP/D95++(d,p)) } & $E=-609.04236$ a.u. \\
\hline $\mathrm{C}$ & -1.660549 & 0.238000 & 0.023941 \\
\hline $\mathrm{C}$ & -0.841590 & 1.510510 & -0.058952 \\
\hline $\mathrm{C}$ & -0.859742 & -1.097609 & -0.072037 \\
\hline
\end{tabular}




$\begin{array}{lrrr}\mathrm{O} & -2.581502 & 0.276860 & -1.073477 \\ \mathrm{~N} & -2.293665 & 0.186628 & 1.333930 \\ \mathrm{C} & 0.507347 & 1.541286 & -0.042265 \\ \mathrm{C} & 0.580023 & -1.001235 & 0.001048 \\ \mathrm{H} & -1.417473 & 2.432873 & -0.120305 \\ \mathrm{O} & -1.512434 & -2.137324 & -0.194357 \\ \mathrm{H} & -2.905817 & -0.635100 & -1.181741 \\ \mathrm{H} & -2.939155 & 0.966474 & 1.447111 \\ \mathrm{H} & -2.809531 & -0.687967 & 1.435118 \\ \mathrm{C} & 1.195483 & 0.257882 & 0.011921 \\ \mathrm{C} & 1.311111 & 2.817663 & -0.088123 \\ \mathrm{C} & 1.610727 & -1.995101 & 0.019531 \\ \mathrm{~N} & 2.547417 & 0.045270 & 0.043315 \\ \mathrm{C} & 2.812234 & -1.320235 & 0.050324 \\ \mathrm{H} & 1.976972 & 2.841533 & -0.962068 \\ \mathrm{H} & 1.935431 & 2.929297 & 0.809613 \\ \mathrm{H} & 0.653192 & 3.689282 & -0.145846 \\ \mathrm{H} & 1.471969 & -3.068025 & 0.015740 \\ \mathrm{H} & 3.829628 & -1.686978 & 0.080470 \\ \mathrm{H} & 3.249753 & 0.772067 & 0.051549 \\ & & & \\ & & & \\ & & & \end{array}$

\section{2}

$\mathrm{C}$

$\mathrm{C}$

$\mathrm{C}$

$\mathrm{O}$

C

$\mathrm{C}$

$\mathrm{H}$

$\mathrm{N}$

$\mathrm{O}$

$\mathrm{C}$

$\mathrm{C}$

$\mathrm{C}$

$\mathrm{H}$

$\mathrm{H}$

$\mathrm{H}$

$\mathrm{N}$

\section{(B3LYP/D95++(d,p)) $\quad E=\mathbf{- 6 0 9 . 0 4 1 0 5}$ a.u.}

$\begin{array}{lll}-1.519446 & 0.927065 & -0.110966\end{array}$

$\begin{array}{lll}-0.427567 & 1.888799 & -0.015038\end{array}$

$\begin{array}{lll}-1.236844 & -0.599353 & 0.056639\end{array}$

$\begin{array}{lll}-2.695787 & 1.252489 & -0.300412\end{array}$

$\begin{array}{lll}0.885559 & 1.501274 & 0.027053\end{array}$

$\begin{array}{lll}0.228699 & -0.924380 & -0.032129\end{array}$

$\begin{array}{lll}-0.705204 & 2.939824 & -0.038971\end{array}$

$\begin{array}{lll}-1.709740 & -0.887664 & 1.405383\end{array}$

$\begin{array}{lll}-1.927056 & -1.303234 & -0.975738\end{array}$

$\begin{array}{lll}1.181413 & 0.088608 & 0.000402\end{array}$

$\begin{array}{lll}2.012272 & 2.500856 & 0.075390\end{array}$

$\begin{array}{lll}0.911846 & -2.164178 & -0.098933\end{array}$

$\begin{array}{lll}-2.697054 & -0.644496 & 1.487141\end{array}$

$\begin{array}{lll}-1.586984 & -1.874801 & 1.623278\end{array}$

$\begin{array}{lll}-2.771156 & -0.831937 & -1.096045\end{array}$

$2.429274 \quad-0.512407 \quad-0.041840$ 


\begin{tabular}{|c|c|c|c|}
\hline $\mathrm{C}$ & 2.273682 & -1.875415 & -0.104903 \\
\hline $\mathrm{H}$ & 0.474423 & -3.152335 & -0.160841 \\
\hline $\mathrm{H}$ & 2.669132 & 2.397001 & -0.799308 \\
\hline $\mathrm{H}$ & 2.629242 & 2.351616 & 0.972039 \\
\hline $\mathrm{H}$ & 1.631040 & 3.524987 & 0.091866 \\
\hline $\mathrm{H}$ & 3.129789 & -2.535449 & -0.155838 \\
\hline $\mathrm{H}$ & 3.315098 & -0.027608 & -0.044015 \\
\hline 3 & \multicolumn{2}{|c|}{ (B3LYP/D95++(d,p)) } & $E=-625.09605$ a.u. \\
\hline $\mathrm{C}$ & -1.640928 & 0.242274 & 0.015623 \\
\hline $\mathrm{C}$ & -0.817223 & 1.517178 & -0.052543 \\
\hline $\mathrm{C}$ & -0.840143 & -1.090120 & -0.057366 \\
\hline $\mathrm{O}$ & -2.532919 & 0.276367 & -1.106337 \\
\hline $\mathrm{N}$ & -2.300612 & 0.188919 & 1.310118 \\
\hline $\mathrm{C}$ & 0.531397 & 1.567611 & -0.032984 \\
\hline $\mathrm{C}$ & 0.584062 & -0.936070 & 0.012519 \\
\hline $\mathrm{H}$ & -1.403945 & 2.432972 & -0.110343 \\
\hline $\mathrm{O}$ & -1.452371 & -2.157965 & -0.162104 \\
\hline $\mathrm{H}$ & -2.894458 & -0.622396 & -1.199262 \\
\hline $\mathrm{H}$ & -2.914508 & 0.991463 & 1.435688 \\
\hline $\mathrm{H}$ & -2.845291 & -0.669332 & 1.395946 \\
\hline $\mathrm{C}$ & 1.245972 & 0.291049 & 0.016108 \\
\hline $\mathrm{C}$ & 1.328876 & 2.844311 & -0.070724 \\
\hline $\mathrm{N}$ & 1.581715 & -1.894192 & 0.020190 \\
\hline $\mathrm{N}$ & 2.605209 & 0.098752 & 0.035745 \\
\hline $\mathrm{C}$ & 2.765161 & -1.221204 & 0.039954 \\
\hline $\mathrm{H}$ & 1.436766 & -2.896216 & 0.017929 \\
\hline $\mathrm{H}$ & 2.006345 & 2.853043 & -0.933129 \\
\hline $\mathrm{H}$ & 1.958268 & 2.932861 & 0.823328 \\
\hline $\mathrm{H}$ & 0.670618 & 3.716476 & -0.127712 \\
\hline $\mathrm{H}$ & 3.721287 & -1.730611 & 0.059197 \\
\hline 4 & \multicolumn{2}{|c|}{$($ B3LYP/D95++(d,p)) } & $E=-625.09549$ a.u. \\
\hline $\mathrm{C}$ & 0.000000 & 0.000000 & 0.000000 \\
\hline $\mathrm{C}$ & 0.000000 & 0.000000 & 1.457400 \\
\hline $\mathrm{C}$ & 1.352231 & 0.000000 & -0.790620 \\
\hline $\mathrm{O}$ & -1.021283 & -0.026852 & -0.692467 \\
\hline $\mathrm{C}$ & 1.133915 & 0.203151 & 2.198190 \\
\hline
\end{tabular}




\begin{tabular}{|c|c|c|c|}
\hline $\mathrm{C}$ & 2.480479 & 0.357440 & 0.122954 \\
\hline $\mathrm{H}$ & -0.970825 & -0.112993 & 1.933795 \\
\hline $\mathrm{N}$ & 1.495542 & -1.374546 & -1.245396 \\
\hline $\mathrm{O}$ & 1.287879 & 0.970069 & -1.835204 \\
\hline $\mathrm{C}$ & 2.392431 & 0.413447 & 1.498117 \\
\hline $\mathrm{C}$ & 1.129435 & 0.251002 & 3.698120 \\
\hline $\mathrm{N}$ & 3.787074 & 0.643695 & -0.176982 \\
\hline $\mathrm{H}$ & 0.666675 & -1.653840 & -1.770157 \\
\hline $\mathrm{H}$ & 2.316552 & -1.482386 & -1.838228 \\
\hline $\mathrm{H}$ & 0.373963 & 0.936205 & -2.172616 \\
\hline $\mathrm{N}$ & 3.623228 & 0.727933 & 2.052404 \\
\hline $\mathrm{C}$ & 4.432533 & 0.864912 & 1.022883 \\
\hline $\mathrm{H}$ & 4.175291 & 0.762583 & -1.104074 \\
\hline $\mathrm{H}$ & 1.526518 & 1.211955 & 4.047210 \\
\hline $\mathrm{H}$ & 1.798534 & -0.520071 & 4.100115 \\
\hline $\mathrm{H}$ & 0.124219 & 0.103997 & 4.102144 \\
\hline $\mathrm{H}$ & 5.482790 & 1.124242 & 1.071659 \\
\hline 5 & \multicolumn{2}{|c|}{ (B3LYP/D95++(d,p)) } & $E=-647.14632$ a.u. \\
\hline $\mathrm{C}$ & 0.000000 & 0.000000 & 0.000000 \\
\hline $\mathrm{C}$ & 0.000000 & 0.000000 & 1.508500 \\
\hline $\mathrm{C}$ & 1.420282 & 0.000000 & -0.610415 \\
\hline $\mathrm{O}$ & -0.693929 & 1.166806 & -0.427894 \\
\hline $\mathrm{N}$ & -0.574918 & -1.256867 & -0.474811 \\
\hline $\mathrm{C}$ & 1.060308 & -0.351039 & 2.260194 \\
\hline $\mathrm{C}$ & 2.526148 & -0.581052 & 0.213569 \\
\hline $\mathrm{H}$ & -0.940519 & 0.293339 & 1.972502 \\
\hline $\mathrm{O}$ & 1.569903 & 0.443891 & -1.738311 \\
\hline $\mathrm{H}$ & -0.342351 & 1.383110 & -1.310649 \\
\hline $\mathrm{H}$ & -0.636198 & -1.252454 & -1.493259 \\
\hline $\mathrm{H}$ & -1.510724 & -1.385704 & -0.093474 \\
\hline $\mathrm{C}$ & 2.336803 & -0.729650 & 1.611677 \\
\hline $\mathrm{C}$ & 0.971867 & -0.374106 & 3.768527 \\
\hline $\mathrm{N}$ & 3.663011 & -0.883172 & -0.430909 \\
\hline $\mathrm{H}$ & 1.729915 & 0.273847 & 4.227534 \\
\hline $\mathrm{H}$ & 1.123823 & -1.387274 & 4.164054 \\
\hline $\mathrm{H}$ & -0.012188 & -0.030354 & 4.099331 \\
\hline $\mathrm{C}$ & 4.678487 & -1.369377 & 0.286473 \\
\hline
\end{tabular}




$\begin{array}{lrrr}\mathrm{C} & 3.433809 & -1.221768 & 2.343232 \\ \mathrm{C} & 4.613612 & -1.551851 & 1.677254 \\ \mathrm{H} & 3.365190 & -1.348194 & 3.419764 \\ \mathrm{H} & 5.584485 & -1.621409 & -0.264463 \\ \mathrm{H} & 5.472873 & -1.940406 & 2.218672\end{array}$

\begin{tabular}{|c|c|c|c|}
\hline 6 & \multicolumn{2}{|c|}{$($ B3LYP/D95++(d,p)) } & $E=-647.15584$ a.u. \\
\hline $\mathrm{C}$ & 1.934293 & 0.480992 & -0.017252 \\
\hline $\mathrm{C}$ & 1.203708 & 1.742788 & -0.054298 \\
\hline $\mathrm{C}$ & 1.155259 & -0.853003 & -0.002398 \\
\hline $\mathrm{O}$ & 3.163999 & 0.421722 & 0.042182 \\
\hline $\mathrm{C}$ & -0.154730 & 1.807065 & -0.024659 \\
\hline $\mathrm{C}$ & -0.360964 & -0.696806 & 0.004058 \\
\hline $\mathrm{H}$ & 1.810993 & 2.644945 & -0.072520 \\
\hline $\mathrm{N}$ & 1.528569 & -1.556195 & -1.206292 \\
\hline $\mathrm{O}$ & 1.492423 & -1.510988 & 1.228851 \\
\hline $\mathrm{C}$ & -0.971387 & 0.578058 & 0.006054 \\
\hline $\mathrm{C}$ & -0.847793 & 3.145939 & -0.027708 \\
\hline $\mathrm{N}$ & -1.047064 & -1.843372 & -0.013262 \\
\hline $\mathrm{H}$ & 2.541167 & -1.651662 & -1.265758 \\
\hline $\mathrm{H}$ & 1.080538 & -2.470854 & -1.223590 \\
\hline $\mathrm{H}$ & 2.455223 & -1.417147 & 1.330176 \\
\hline $\mathrm{C}$ & -2.384289 & -1.786521 & 0.000293 \\
\hline $\mathrm{H}$ & -1.470120 & 3.271400 & 0.867837 \\
\hline $\mathrm{H}$ & -1.507810 & 3.247508 & -0.898980 \\
\hline $\mathrm{H}$ & -0.119561 & 3.960230 & -0.054228 \\
\hline $\mathrm{C}$ & -2.378260 & 0.612533 & 0.024748 \\
\hline $\mathrm{H}$ & -2.908146 & -2.742241 & -0.013939 \\
\hline $\mathrm{C}$ & -3.097955 & -0.582127 & 0.028559 \\
\hline $\mathrm{H}$ & -2.906790 & 1.561395 & 0.031558 \\
\hline $\mathrm{H}$ & -4.184698 & -0.585976 & 0.043684 \\
\hline 7 & \multicolumn{2}{|c|}{ (B3LYP/D95++(d,p)) } & $E=-647.15548$ a.u. \\
\hline $\mathrm{C}$ & -1.896315 & 0.016608 & 0.057983 \\
\hline $\mathrm{C}$ & -1.299717 & 1.396681 & -0.066306 \\
\hline $\mathrm{C}$ & -0.874776 & -1.125474 & -0.135361 \\
\hline $\mathrm{O}$ & -2.907336 & -0.093349 & -0.944738 \\
\hline $\mathrm{N}$ & -2.371972 & -0.172303 & 1.422947 \\
\hline
\end{tabular}




\begin{tabular}{|c|c|c|c|}
\hline $\mathrm{C}$ & 0.020780 & 1.660896 & -0.077277 \\
\hline $\mathrm{C}$ & 0.563087 & -0.803092 & -0.034361 \\
\hline $\mathrm{H}$ & -2.025876 & 2.206015 & -0.131688 \\
\hline $\mathrm{O}$ & -1.302496 & -2.258706 & -0.340168 \\
\hline $\mathrm{H}$ & -3.070690 & -1.044728 & -1.069224 \\
\hline $\mathrm{H}$ & -2.814169 & -1.086399 & 1.520512 \\
\hline $\mathrm{H}$ & -3.045371 & 0.550187 & 1.671671 \\
\hline $\mathrm{C}$ & 0.995593 & 0.547375 & -0.009968 \\
\hline $\mathrm{C}$ & 0.558927 & 3.066773 & -0.157875 \\
\hline $\mathrm{C}$ & 1.518352 & -1.827523 & -0.009125 \\
\hline $\mathrm{H}$ & 1.213926 & 3.186336 & -1.028396 \\
\hline $\mathrm{H}$ & 1.169543 & 3.299515 & 0.721960 \\
\hline $\mathrm{H}$ & -0.260659 & 3.788720 & -0.225373 \\
\hline $\mathrm{C}$ & 2.867725 & -1.485762 & 0.063026 \\
\hline $\mathrm{N}$ & 2.302830 & 0.870541 & 0.047808 \\
\hline $\mathrm{H}$ & 1.184482 & -2.861766 & -0.042334 \\
\hline $\mathrm{C}$ & 3.199515 & -0.121748 & 0.090115 \\
\hline $\mathrm{H}$ & 3.647623 & -2.241703 & 0.096096 \\
\hline $\mathrm{H}$ & 4.244821 & 0.184190 & 0.145619 \\
\hline 8 & \multicolumn{2}{|c|}{ (B3LYP/D95++(d,p)) } & $E=-647.15880$ a.u. \\
\hline $\mathrm{C}$ & -1.910603 & 0.511116 & -0.194466 \\
\hline $\mathrm{C}$ & -1.160302 & 1.755239 & -0.079875 \\
\hline $\mathrm{C}$ & -1.187016 & -0.824304 & 0.103846 \\
\hline $\mathrm{O}$ & -3.110935 & 0.456303 & -0.466540 \\
\hline $\mathrm{C}$ & 0.196534 & 1.779572 & 0.022183 \\
\hline $\mathrm{C}$ & 0.322504 & -0.732121 & -0.017668 \\
\hline $\mathrm{H}$ & -1.739476 & 2.674461 & -0.133606 \\
\hline $\mathrm{N}$ & -1.512194 & -1.083566 & 1.503424 \\
\hline $\mathrm{O}$ & -1.652731 & -1.816107 & -0.805222 \\
\hline $\mathrm{C}$ & 0.975318 & 0.517952 & 0.005335 \\
\hline $\mathrm{C}$ & 0.953949 & 3.074228 & 0.104026 \\
\hline $\mathrm{C}$ & 1.101920 & -1.887287 & -0.110045 \\
\hline $\mathrm{H}$ & -2.523340 & -1.143253 & 1.622609 \\
\hline $\mathrm{H}$ & -1.089698 & -1.956083 & 1.815323 \\
\hline $\mathrm{H}$ & -2.585245 & -1.594041 & -0.981413 \\
\hline $\mathrm{C}$ & 2.493904 & -1.765350 & -0.147911 \\
\hline $\mathrm{H}$ & 1.645227 & 3.169278 & -0.741055 \\
\hline
\end{tabular}




\begin{tabular}{|c|c|c|c|}
\hline $\mathrm{H}$ & 1.576645 & 3.094364 & 1.006034 \\
\hline $\mathrm{H}$ & 0.270219 & 3.927518 & 0.114023 \\
\hline $\mathrm{N}$ & 2.318345 & 0.635123 & -0.020123 \\
\hline $\mathrm{H}$ & 0.621428 & -2.860552 & -0.171890 \\
\hline $\mathrm{H}$ & 3.135713 & -2.639330 & -0.224686 \\
\hline $\mathrm{C}$ & 3.050853 & -0.480518 & -0.087362 \\
\hline $\mathrm{H}$ & 4.132445 & -0.345993 & -0.102724 \\
\hline 9 & \multicolumn{2}{|c|}{ (B3LYP/D95++(d,p)) } & $E=-663.18811$ a.u. \\
\hline $\mathrm{C}$ & -1.881011 & -0.008679 & 0.110875 \\
\hline $\mathrm{C}$ & -1.316993 & 1.381659 & -0.069980 \\
\hline $\mathrm{C}$ & -0.867751 & -1.123313 & -0.233449 \\
\hline $\mathrm{O}$ & -3.017317 & -0.122756 & -0.734937 \\
\hline $\mathrm{N}$ & -2.147468 & -0.231115 & 1.529947 \\
\hline $\mathrm{C}$ & -0.000557 & 1.661957 & -0.116537 \\
\hline $\mathrm{C}$ & 0.587718 & -0.794278 & -0.059534 \\
\hline $\mathrm{H}$ & -2.059542 & 2.174438 & -0.145489 \\
\hline $\mathrm{O}$ & -1.274968 & -2.211753 & -0.600480 \\
\hline $\mathrm{H}$ & -3.109774 & -1.068369 & -0.949999 \\
\hline $\mathrm{H}$ & -2.604470 & -1.132495 & 1.668942 \\
\hline $\mathrm{H}$ & -2.749044 & 0.501502 & 1.902364 \\
\hline $\mathrm{C}$ & 0.982795 & 0.561485 & -0.029672 \\
\hline $\mathrm{C}$ & 0.500148 & 3.081161 & -0.248132 \\
\hline $\mathrm{N}$ & 1.457711 & -1.810042 & 0.002598 \\
\hline $\mathrm{H}$ & 1.142421 & 3.201756 & -1.130123 \\
\hline $\mathrm{H}$ & 1.089538 & 3.380208 & 0.628703 \\
\hline $\mathrm{H}$ & -0.338809 & 3.776285 & -0.341700 \\
\hline $\mathrm{C}$ & 2.747333 & -1.477529 & 0.110870 \\
\hline $\mathrm{C}$ & 2.373871 & 0.767275 & 0.057324 \\
\hline $\mathrm{N}$ & 3.256225 & -0.234702 & 0.134512 \\
\hline $\mathrm{H}$ & 2.789082 & 1.773330 & 0.067899 \\
\hline $\mathrm{H}$ & 3.460289 & -2.297994 & 0.182758 \\
\hline 10 & \multicolumn{2}{|c|}{ (B3LYP/D95++(d,p)) } & $E=-663.20000$ a.u. \\
\hline $\mathrm{C}$ & -1.915267 & 0.519180 & 0.010773 \\
\hline $\mathrm{C}$ & -1.153833 & 1.764895 & 0.048821 \\
\hline $\mathrm{C}$ & -1.172429 & -0.837859 & 0.003806 \\
\hline $\mathrm{O}$ & -3.144807 & 0.489611 & -0.052050 \\
\hline
\end{tabular}




\begin{tabular}{|c|c|c|c|}
\hline $\mathrm{C}$ & 0.205430 & 1.799702 & 0.023128 \\
\hline $\mathrm{C}$ & 0.345285 & -0.711412 & -0.006425 \\
\hline $\mathrm{H}$ & -1.741026 & 2.680252 & 0.065729 \\
\hline $\mathrm{N}$ & -1.549273 & -1.516428 & 1.219143 \\
\hline $\mathrm{O}$ & -1.520931 & -1.500572 & -1.217714 \\
\hline $\mathrm{C}$ & 0.980962 & 0.546789 & -0.006770 \\
\hline $\mathrm{C}$ & 0.940222 & 3.115441 & 0.028294 \\
\hline $\mathrm{N}$ & 1.034448 & -1.855707 & 0.006733 \\
\hline $\mathrm{H}$ & -2.563182 & -1.569371 & 1.302857 \\
\hline $\mathrm{H}$ & -1.137656 & -2.447882 & 1.242722 \\
\hline $\mathrm{H}$ & -2.480181 & -1.382580 & -1.328042 \\
\hline $\mathrm{C}$ & 2.368919 & -1.757116 & -0.006193 \\
\hline $\mathrm{H}$ & 1.567505 & 3.221286 & -0.866254 \\
\hline $\mathrm{H}$ & 1.603072 & 3.194005 & 0.899722 \\
\hline $\mathrm{H}$ & 0.238049 & 3.952240 & 0.055249 \\
\hline $\mathrm{C}$ & 2.386861 & 0.510960 & -0.023084 \\
\hline $\mathrm{H}$ & 2.926943 & -2.692893 & 0.002653 \\
\hline $\mathrm{N}$ & 3.089978 & -0.628032 & -0.028872 \\
\hline $\mathrm{H}$ & 2.969411 & 1.430313 & -0.029631 \\
\hline 11 & \multicolumn{2}{|c|}{ (B3LYP/D95++(d,p)) } & $E=-739.43816$ a.u. \\
\hline $\mathrm{C}$ & 2.968439 & -1.910633 & -0.019750 \\
\hline $\mathrm{N}$ & 2.967880 & -0.545325 & -0.046121 \\
\hline $\mathrm{C}$ & 1.664907 & -0.096345 & -0.039024 \\
\hline $\mathrm{C}$ & 0.816506 & -1.208078 & -0.004024 \\
\hline $\mathrm{C}$ & 1.649712 & -2.361042 & 0.007530 \\
\hline $\mathrm{C}$ & -0.634653 & -1.036631 & 0.012087 \\
\hline $\mathrm{C}$ & -1.170988 & 0.272170 & 0.001484 \\
\hline $\mathrm{C}$ & 1.243496 & 1.276954 & -0.027315 \\
\hline $\mathrm{C}$ & -0.286407 & 1.527521 & 0.008566 \\
\hline $\mathrm{N}$ & -2.483750 & 0.521428 & -0.030368 \\
\hline $\mathrm{C}$ & -1.540064 & -2.109946 & 0.019224 \\
\hline $\mathrm{C}$ & -2.910248 & -1.849160 & 0.008184 \\
\hline $\mathrm{N}$ & -0.598857 & 2.303399 & -1.166382 \\
\hline $\mathrm{H}$ & 3.893093 & -2.473622 & -0.023025 \\
\hline $\mathrm{H}$ & 1.347062 & -3.399864 & 0.032949 \\
\hline $\mathrm{H}$ & -1.173128 & -3.133861 & 0.028896 \\
\hline $\mathrm{H}$ & -3.638813 & -2.655758 & 0.014909 \\
\hline
\end{tabular}




$\begin{array}{lrcc}\mathrm{O} & 2.010662 & 2.246345 & -0.000174 \\ \mathrm{O} & -0.549846 & 2.178143 & 1.265741 \\ \mathrm{H} & -0.027147 & 3.145536 & -1.204295 \\ \mathrm{H} & -1.589653 & 2.539810 & -1.166252 \\ \mathrm{H} & 0.118997 & 2.877920 & 1.356518 \\ \mathrm{C} & -3.332693 & -0.513657 & -0.025077 \\ \mathrm{H} & 3.772292 & 0.068361 & -0.064891 \\ \mathrm{H} & -4.394186 & -0.267864 & -0.050563\end{array}$

12

C

$\mathrm{N}$

C

C

C

C

C

C

C

$\mathrm{N}$

C

C

$\mathrm{O}$

$\mathrm{N}$

$\mathrm{O}$

$\mathrm{H}$

$\mathrm{H}$

$\mathrm{H}$

$\mathrm{H}$

$\mathrm{H}$

$\mathrm{H}$

$\mathrm{H}$

C

$\mathrm{H}$

$\mathrm{H}$

13

C
$($ B3LYP/D95++(d,p) $\quad$ E= $=\mathbf{- 7 3 9 . 4 2 5 2 3}$ a.u.

$\begin{array}{lll}0.000000 & 0.000000 & 0.000000\end{array}$

$\begin{array}{lll}0.000000 & 0.000000 & 1.383047\end{array}$

$\begin{array}{lll}1.292230 & 0.000000 & 1.833361\end{array}$

$\begin{array}{lll}2.141553 & -0.011266 & 0.739997\end{array}$

$\begin{array}{lll}1.313282 & -0.018689 & -0.432196\end{array}$

$\begin{array}{lll}3.589065 & 0.007415 & 0.924485\end{array}$

$\begin{array}{lll}4.126885 & 0.202395 & 2.225910\end{array}$

$\begin{array}{lll}1.725300 & -0.060782 & 3.264195\end{array}$

$3.203369 \quad 0.414818 \quad 3.390532$

$\begin{array}{lll}5.441554 & 0.260567 & 2.492053\end{array}$

$\begin{array}{lll}4.510642 & -0.120262 & -0.130802\end{array}$

$\begin{array}{lll}5.874435 & -0.073773 & 0.145942\end{array}$

$\begin{array}{lll}3.545537 & 0.905740 & 4.455069\end{array}$

$\begin{array}{lll}1.779292 & -1.451577 & 3.708728\end{array}$

$\begin{array}{lll}0.881869 & 0.775036 & 4.046988\end{array}$

$\begin{array}{lll}-0.925547 & 0.024011 & -0.559309\end{array}$

$\begin{array}{lll}1.631328 & -0.016714 & -1.467060\end{array}$

$\begin{array}{lll}4.156494 & -0.261315 & -1.149296\end{array}$

$6.611084 \quad-0.177742 \quad-0.647285$

$\begin{array}{lll}0.878002 & -1.909157 & 3.586235\end{array}$

$2.042661 \quad-1.497458 \quad 4.692826$

$\begin{array}{lll}1.439729 & 1.111630 & 4.773110\end{array}$

$\begin{array}{lll}6.293222 & 0.114046 & 1.475969\end{array}$

$\begin{array}{lll}-0.810185 & 0.107489 & 1.978731\end{array}$

$\begin{array}{lll}7.354213 & 0.149651 & 1.722493\end{array}$

(B3LYP/D95++(d,p)) $\quad E=\mathbf{- 7 5 5 . 4 8 2 6 7}$ a.u.

$\begin{array}{lll}0.000000 & 0.000000 \quad 0.000000\end{array}$ 


\begin{tabular}{|c|c|c|c|}
\hline $\mathrm{N}$ & 0.000000 & 0.000000 & 1.365280 \\
\hline $\mathrm{C}$ & 1.302129 & 0.000000 & 1.815345 \\
\hline $\mathrm{C}$ & 2.149364 & 0.003797 & 0.701520 \\
\hline $\mathrm{C}$ & 1.318701 & 0.003227 & -0.451976 \\
\hline $\mathrm{C}$ & 3.597215 & 0.006948 & 0.883245 \\
\hline $\mathrm{C}$ & 4.133533 & 0.016392 & 2.188079 \\
\hline $\mathrm{C}$ & 1.723648 & 0.030671 & 3.189361 \\
\hline $\mathrm{C}$ & 3.254529 & 0.049222 & 3.445473 \\
\hline $\mathrm{N}$ & 5.451152 & -0.021077 & 2.406458 \\
\hline $\mathrm{C}$ & 4.533366 & -0.014843 & -0.162262 \\
\hline $\mathrm{N}$ & 5.855276 & -0.032129 & 0.046176 \\
\hline $\mathrm{N}$ & 3.552815 & -1.125961 & 4.224015 \\
\hline $\mathrm{H}$ & -0.924470 & -0.002683 & -0.563175 \\
\hline $\mathrm{H}$ & 1.622602 & 0.005272 & -1.490566 \\
\hline $\mathrm{H}$ & 4.205343 & -0.018699 & -1.201493 \\
\hline $\mathrm{O}$ & 0.957693 & 0.082636 & 4.157694 \\
\hline $\mathrm{O}$ & 3.542268 & 1.306338 & 4.078924 \\
\hline $\mathrm{H}$ & 2.965988 & -1.167458 & 5.055386 \\
\hline $\mathrm{H}$ & 4.539352 & -1.134668 & 4.477055 \\
\hline $\mathrm{H}$ & 2.872666 & 1.424166 & 4.773968 \\
\hline $\mathrm{C}$ & 6.244578 & -0.042319 & 1.328679 \\
\hline $\mathrm{H}$ & -0.805604 & 0.001960 & 1.977998 \\
\hline $\mathrm{H}$ & 7.317746 & -0.070071 & 1.513984 \\
\hline 14 & \multicolumn{2}{|c|}{ (B3LYP/D95++(d,p)) } & $E=-755.46699$ a.u. \\
\hline $\mathrm{C}$ & 2.381511 & 2.390179 & -0.193552 \\
\hline $\mathrm{N}$ & 2.636354 & 1.034142 & -0.097252 \\
\hline $\mathrm{C}$ & 1.450356 & 0.357554 & -0.012954 \\
\hline $\mathrm{C}$ & 0.415285 & 1.278110 & -0.045979 \\
\hline $\mathrm{C}$ & 1.012262 & 2.578314 & -0.155110 \\
\hline $\mathrm{C}$ & -0.967988 & 0.823331 & 0.000999 \\
\hline $\mathrm{C}$ & -1.261193 & -0.558733 & -0.082654 \\
\hline $\mathrm{C}$ & 1.291693 & -1.121666 & 0.165162 \\
\hline $\mathrm{C}$ & -0.141469 & -1.552052 & -0.264077 \\
\hline $\mathrm{N}$ & -2.513156 & -1.036360 & -0.064956 \\
\hline $\mathrm{C}$ & -2.093526 & 1.665809 & 0.094798 \\
\hline $\mathrm{N}$ & -3.345008 & 1.201832 & 0.124209 \\
\hline $\mathrm{O}$ & -0.300559 & -2.680756 & -0.694823 \\
\hline
\end{tabular}




\begin{tabular}{|c|c|c|c|}
\hline $\mathrm{N}$ & 1.322292 & -1.455687 & 1.586648 \\
\hline $\mathrm{O}$ & 2.259931 & -1.797447 & -0.624333 \\
\hline $\mathrm{H}$ & 3.187633 & 3.104532 & -0.292808 \\
\hline $\mathrm{H}$ & 0.508553 & 3.534461 & -0.216701 \\
\hline $\mathrm{H}$ & -1.972014 & 2.747274 & 0.151531 \\
\hline $\mathrm{H}$ & 2.166990 & -1.102579 & 2.031604 \\
\hline $\mathrm{H}$ & 1.271950 & -2.465505 & 1.718158 \\
\hline $\mathrm{H}$ & 1.848062 & -2.635660 & -0.904823 \\
\hline $\mathrm{C}$ & -3.490224 & -0.133837 & 0.049225 \\
\hline $\mathrm{H}$ & 3.539886 & 0.589306 & -0.191643 \\
\hline $\mathrm{H}$ & -4.509661 & -0.515759 & 0.082241 \\
\hline 15 & \multicolumn{2}{|c|}{ (B3LYP/D95++(d,p)) } & $E=-739.43376$ a.u. \\
\hline $\mathrm{C}$ & 0.000000 & 0.000000 & 0.000000 \\
\hline $\mathrm{C}$ & 0.000000 & 0.000000 & 1.377959 \\
\hline $\mathrm{C}$ & 1.371329 & 0.000000 & 1.788103 \\
\hline $\mathrm{C}$ & 2.166020 & 0.003471 & 0.637125 \\
\hline $\mathrm{N}$ & 1.318423 & 0.003145 & -0.438705 \\
\hline $\mathrm{C}$ & 3.618874 & 0.007151 & 0.623427 \\
\hline $\mathrm{C}$ & 4.285714 & 0.005634 & 1.871346 \\
\hline $\mathrm{C}$ & 1.970325 & 0.032828 & 3.107373 \\
\hline $\mathrm{C}$ & 3.524107 & 0.027845 & 3.201443 \\
\hline $\mathrm{N}$ & 5.616095 & -0.035392 & 1.981398 \\
\hline $\mathrm{C}$ & 4.406411 & -0.004253 & -0.540299 \\
\hline $\mathrm{C}$ & 5.795765 & -0.024250 & -0.421755 \\
\hline $\mathrm{N}$ & 3.878168 & -1.165705 & 3.932633 \\
\hline $\mathrm{H}$ & 3.948019 & -0.001040 & -1.527928 \\
\hline $\mathrm{H}$ & 6.436557 & -0.031704 & -1.299406 \\
\hline $\mathrm{O}$ & 1.342064 & 0.102775 & 4.164902 \\
\hline $\mathrm{O}$ & 3.889002 & 1.266817 & 3.833418 \\
\hline $\mathrm{H}$ & 3.372972 & -1.202595 & 4.816584 \\
\hline $\mathrm{H}$ & 4.883619 & -1.182218 & 4.095353 \\
\hline $\mathrm{H}$ & 3.269608 & 1.382046 & 4.574447 \\
\hline $\mathrm{C}$ & 6.353312 & -0.047591 & 0.863848 \\
\hline $\mathrm{H}$ & 7.434311 & -0.079520 & 0.998030 \\
\hline $\mathrm{H}$ & -0.818041 & -0.003148 & -0.708118 \\
\hline $\mathrm{H}$ & -0.865204 & -0.000268 & 2.027158 \\
\hline $\mathrm{H}$ & 1.601541 & 0.006594 & -1.408472 \\
\hline
\end{tabular}




\begin{tabular}{|c|c|c|c|}
\hline 16 & \multicolumn{2}{|c|}{ (B3LYP/D95++(d,p)) } & $E=-739.42254$ a.u. \\
\hline $\mathrm{C}$ & 2.294471 & 2.477842 & -0.174924 \\
\hline $\mathrm{C}$ & 2.703080 & 1.152174 & -0.106251 \\
\hline $\mathrm{C}$ & 1.524130 & 0.363759 & -0.025696 \\
\hline $\mathrm{C}$ & 0.432190 & 1.219879 & -0.044390 \\
\hline $\mathrm{N}$ & 0.919282 & 2.511356 & -0.130491 \\
\hline $\mathrm{C}$ & -0.953022 & 0.797305 & 0.001290 \\
\hline $\mathrm{C}$ & -1.230717 & -0.595832 & -0.075373 \\
\hline $\mathrm{C}$ & 1.346793 & -1.120170 & 0.135828 \\
\hline $\mathrm{C}$ & -0.097349 & -1.572074 & -0.233945 \\
\hline $\mathrm{N}$ & -2.469484 & -1.107314 & -0.062538 \\
\hline $\mathrm{C}$ & -2.056885 & 1.666855 & 0.091634 \\
\hline $\mathrm{C}$ & -3.342313 & 1.131904 & 0.114045 \\
\hline $\mathrm{O}$ & -0.256960 & -2.725664 & -0.601793 \\
\hline $\mathrm{N}$ & 1.439220 & -1.464003 & 1.555706 \\
\hline $\mathrm{O}$ & 2.274842 & -1.800903 & -0.695555 \\
\hline $\mathrm{H}$ & 2.873275 & 3.388016 & -0.261962 \\
\hline $\mathrm{H}$ & 0.359666 & 3.348551 & -0.201732 \\
\hline $\mathrm{H}$ & -1.915798 & 2.744550 & 0.153175 \\
\hline $\mathrm{H}$ & -4.215112 & 1.775883 & 0.189320 \\
\hline $\mathrm{H}$ & 2.317056 & -1.124949 & 1.945078 \\
\hline $\mathrm{H}$ & 1.393604 & -2.475906 & 1.675302 \\
\hline $\mathrm{H}$ & 1.861238 & -2.656798 & -0.911373 \\
\hline $\mathrm{C}$ & -3.499049 & -0.263870 & 0.039219 \\
\hline $\mathrm{H}$ & -4.492815 & -0.710465 & 0.060604 \\
\hline $\mathrm{H}$ & 3.724720 & 0.796749 & -0.136980 \\
\hline
\end{tabular}

\section{Transition States}

\begin{tabular}{llrc} 
6MIQ-TS & \multicolumn{2}{l}{$($ B3LYP/D95++(d,p)) } & $\mathbf{E}=\mathbf{- 6 4 8 . 3 0 5 8 1}$ a.u. \\
C & 0.000000 & 0.000000 & 0.000000 \\
N & 0.000000 & 0.000000 & 1.365046 \\
C & 1.301757 & 0.000000 & 1.807692 \\
C & 2.154287 & -0.016476 & 0.692922 \\
C & 1.316694 & -0.017522 & -0.468585 \\
C & 3.615366 & -0.063139 & 0.887389 \\
C & 4.101270 & -0.032767 & 2.149802
\end{tabular}




$\begin{array}{lrrc}\mathrm{C} & 1.731659 & -0.064341 & 3.178500 \\ \mathrm{C} & 3.248622 & 0.203505 & 3.377646 \\ \mathrm{~N} & 3.697517 & -0.654032 & 4.594623 \\ \mathrm{O} & 0.992879 & -0.299816 & 4.141897 \\ \mathrm{O} & 3.492938 & 1.420472 & 3.968855 \\ \mathrm{C} & 1.685822 & -0.029440 & -1.929412 \\ \mathrm{C} & 4.538986 & -0.107023 & -0.306460 \\ \mathrm{H} & -0.924408 & 0.014252 & -0.563981 \\ \mathrm{H} & -0.803873 & 0.009542 & 1.979049 \\ \mathrm{H} & 5.177031 & 0.016923 & 2.318730 \\ \mathrm{H} & 2.879322 & -1.042317 & 5.076861 \\ \mathrm{H} & 4.428012 & -1.352022 & 4.441719 \\ \mathrm{H} & 2.259702 & -0.923194 & -2.202271 \\ \mathrm{H} & 2.287233 & 0.844221 & -2.207438 \\ \mathrm{H} & 0.782102 & -0.017161 & -2.548401 \\ \mathrm{H} & 4.413288 & 0.781265 & -0.938511 \\ \mathrm{H} & 5.584060 & -0.146583 & 0.015466 \\ \mathrm{H} & 4.341864 & -0.983108 & -0.936854 \\ \mathrm{H} & 3.927075 & 0.468038 & 4.912837 \\ & & & \end{array}$

\begin{tabular}{|c|c|c|c|}
\hline 7MIQ-TS & \multicolumn{2}{|c|}{ (B3LYP/D95++(d,p)) } & $E=-648.30196$ a.u. \\
\hline $\mathrm{C}$ & 0.000000 & 0.000000 & 0.000000 \\
\hline $\mathrm{C}$ & 0.000000 & 0.000000 & 1.392509 \\
\hline $\mathrm{C}$ & 1.273725 & 0.000000 & 2.110522 \\
\hline $\mathrm{C}$ & 2.450132 & 0.002061 & 1.409300 \\
\hline $\mathrm{C}$ & 2.523047 & 0.096470 & -0.045376 \\
\hline $\mathrm{C}$ & 1.201703 & -0.020024 & -0.890646 \\
\hline $\mathrm{N}$ & -1.285989 & -0.136264 & -0.434809 \\
\hline $\mathrm{C}$ & -2.130481 & -0.211389 & 0.657255 \\
\hline $\mathrm{C}$ & -1.378916 & -0.121050 & 1.816564 \\
\hline $\mathrm{O}$ & 3.573445 & 0.325796 & -0.648203 \\
\hline $\mathrm{N}$ & 1.259669 & 1.212332 & -1.882131 \\
\hline $\mathrm{O}$ & 1.172860 & -0.966145 & -1.871640 \\
\hline $\mathrm{C}$ & 1.293764 & -0.023203 & 3.616900 \\
\hline $\mathrm{C}$ & -1.958554 & -0.161801 & 3.205633 \\
\hline $\mathrm{H}$ & 3.406644 & 0.009544 & 1.927078 \\
\hline $\mathrm{H}$ & -1.529260 & -0.350835 & -1.393619 \\
\hline $\mathrm{H}$ & -3.200092 & -0.319541 & 0.533033 \\
\hline
\end{tabular}




$\begin{array}{lrrr}\mathrm{H} & 0.531409 & 1.925979 & -1.814028 \\ \mathrm{H} & 2.200474 & 1.618722 & -1.897434 \\ \mathrm{H} & 2.318716 & -0.003043 & 3.996913 \\ \mathrm{H} & 0.801637 & -0.926437 & 3.998611 \\ \mathrm{H} & 0.750326 & 0.835056 & 4.031233 \\ \mathrm{H} & -1.715596 & 0.740004 & 3.781324 \\ \mathrm{H} & -1.594643 & -1.023634 & 3.778597 \\ \mathrm{H} & -3.050199 & -0.237590 & 3.157186 \\ \mathrm{H} & 1.173811 & 0.241912 & -2.571073\end{array}$

\section{IIQ-TS}

$\mathrm{H}$

C

$\mathrm{N}$

C

C

C

C

C

C

C

$\mathrm{N}$

O

$\mathrm{O}$

C

C

C

C

C

$\mathrm{N}$

C

C

C

C

C

$\mathrm{H}$

$\mathrm{H}$

$\mathrm{H}$

C

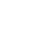

C

C

C

C

C

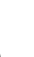

O

C

C

C

C

$\mathrm{N}$

C

C

C

C

H

\section{(B3LYP/D95 (d,p)) $\quad E=\mathbf{- 1 0 1 0 . 9 5 9 7 2 ~ a . u . ~}$}

$\begin{array}{lll}2.633893 & 0.958366 & -3.777163\end{array}$

$\begin{array}{lll}2.740994 & 0.432275 & 2.511168\end{array}$

$\begin{array}{lll}3.575080 & 0.618799 & 1.444727\end{array}$

$\begin{array}{lll}2.839382 & 0.536518 & 0.285470\end{array}$

$\begin{array}{lll}1.501798 & 0.276636 & 0.626560\end{array}$

$\begin{array}{lll}1.438339 & 0.206228 & 2.054363\end{array}$

$\begin{array}{lll}0.503458 & 0.065645 & -0.437852\end{array}$

$\begin{array}{lll}0.897185 & 0.143119 & -1.733155\end{array}$

$3.359258 \quad 0.537101 \quad-1.055460$

$\begin{array}{lll}2.270506 & 0.621370 & -2.156458\end{array}$

$\begin{array}{lll}2.825415 & -0.144997 & -3.390466\end{array}$

$\begin{array}{lll}4.559807 & 0.485274 & -1.351968\end{array}$

$\begin{array}{lll}2.221787 & 1.850657 & -2.760397\end{array}$

$\begin{array}{lll}0.258656 & -0.072632 & 2.947197\end{array}$

$\begin{array}{lll}-0.919473 & -0.176373 & -0.103050\end{array}$

$\begin{array}{lll}-1.684957 & -1.325020 & -0.186338\end{array}$

$\begin{array}{lll}-3.008150 & -0.972228 & 0.277679\end{array}$

$\begin{array}{lll}-2.985867 & 0.409752 & 0.623885\end{array}$

$\begin{array}{lll}-1.699183 & 0.864493 & 0.402993\end{array}$

$\begin{array}{lll}-4.207664 & -1.697779 & 0.430362\end{array}$

$\begin{array}{lll}-5.341846 & -1.036322 & 0.905473\end{array}$

$\begin{array}{lll}-5.300017 & 0.340435 & 1.232095\end{array}$

$\begin{array}{lll}-4.122958 & 1.082109 & 1.096376\end{array}$

$\begin{array}{lll}-1.239635 & -2.688647 & -0.632507\end{array}$

$\begin{array}{lll}3.108805 & 0.466229 & 3.528792\end{array}$

$\begin{array}{lll}4.571979 & 0.787244 & 1.467243\end{array}$

$\begin{array}{lll}0.156254 & 0.070377 & -2.527570\end{array}$ 


$\begin{array}{lrrr}\mathrm{H} & 3.822515 & -0.343172 & -3.247976 \\ \mathrm{H} & 2.307680 & -0.957673 & -3.729975 \\ \mathrm{H} & -0.480498 & 0.735201 & 2.910561 \\ \mathrm{H} & 0.583680 & -0.186444 & 3.986793 \\ \mathrm{H} & -0.259874 & -0.990371 & 2.649794 \\ \mathrm{H} & -1.402842 & 1.829983 & 0.421895 \\ \mathrm{H} & -4.251581 & -2.755641 & 0.178102 \\ \mathrm{H} & -6.275552 & -1.581829 & 1.026772 \\ \mathrm{H} & -6.200545 & 0.830360 & 1.596925 \\ \mathrm{H} & -4.094465 & 2.140110 & 1.350069 \\ \mathrm{H} & -1.810088 & -3.028357 & -1.507163 \\ \mathrm{H} & -0.179095 & -2.686868 & -0.902278 \\ \mathrm{H} & -1.386279 & -3.436146 & 0.158473\end{array}$

\begin{tabular}{lrrc} 
7IIQ-TS & \multicolumn{2}{c}{$(\mathbf{B 3 L Y P} / \mathbf{D} 95(\mathbf{d}, \mathbf{p}))$} & $\mathbf{E}=\mathbf{- 1 0 1 0 . 9 5 4 4 4} \mathbf{a . u .}$ \\
$\mathrm{H}$ & -1.868712 & 0.085570 & -4.866336 \\
$\mathrm{C}$ & -1.894772 & 0.047098 & -2.038002 \\
$\mathrm{C}$ & -1.341006 & 0.085398 & -0.758672 \\
$\mathrm{C}$ & 0.118148 & 0.105574 & -0.618164 \\
$\mathrm{C}$ & 0.914125 & 0.193789 & -1.734447 \\
$\mathrm{C}$ & 0.378048 & 0.307084 & -3.086177 \\
$\mathrm{C}$ & -1.141853 & 0.005607 & -3.332508 \\
$\mathrm{~N}$ & -3.251616 & -0.042135 & -1.920526 \\
$\mathrm{C}$ & -3.596733 & -0.026644 & -0.580316 \\
$\mathrm{C}$ & -2.444816 & 0.071004 & 0.182363 \\
$\mathrm{O}$ & 1.068529 & 0.636812 & -4.054732 \\
$\mathrm{~N}$ & -1.604338 & 1.120625 & -4.350357 \\
$\mathrm{O}$ & -1.435799 & -1.042747 & -4.148085 \\
$\mathrm{C}$ & 0.762112 & -0.017508 & 0.699905 \\
$\mathrm{C}$ & -2.442373 & 0.203563 & 1.683751 \\
$\mathrm{C}$ & 1.740647 & 0.761077 & 1.303344 \\
$\mathrm{C}$ & 2.022722 & 0.145905 & 2.577693 \\
$\mathrm{C}$ & 1.201321 & -1.013157 & 2.687993 \\
$\mathrm{~N}$ & 0.426955 & -1.075072 & 1.547258 \\
$\mathrm{C}$ & 2.901431 & 0.468098 & 3.633949 \\
$\mathrm{C}$ & 2.951261 & -0.369340 & 4.748411 \\
$\mathrm{C}$ & 2.135934 & -1.525143 & 4.830312 \\
$\mathrm{C}$ & 1.251467 & -1.863834 & 3.803447 \\
& & & \\
$\mathrm{~N}$ & &
\end{tabular}




$\begin{array}{lrrr}\mathrm{C} & 2.362787 & 2.028914 & 0.790190 \\ \mathrm{H} & 1.996333 & 0.194797 & -1.644430 \\ \mathrm{H} & -3.868368 & -0.267538 & -2.689787 \\ \mathrm{H} & -4.631146 & -0.061728 & -0.265683 \\ \mathrm{H} & -2.283150 & 1.824437 & -4.053282 \\ \mathrm{H} & -0.768336 & 1.539907 & -4.773347 \\ \mathrm{H} & -1.756282 & 0.989866 & 2.016140 \\ \mathrm{H} & -2.137292 & -0.718813 & 2.189916 \\ \mathrm{H} & -3.446830 & 0.458841 & 2.037940 \\ \mathrm{H} & -0.147721 & -1.857389 & 1.268836 \\ \mathrm{H} & 3.536434 & 1.349988 & 3.577336 \\ \mathrm{H} & 3.626714 & -0.138381 & 5.569529 \\ \mathrm{H} & 2.198875 & -2.160589 & 5.711347 \\ \mathrm{H} & 0.625110 & -2.751093 & 3.872261 \\ \mathrm{H} & 3.432248 & 1.891429 & 0.582515 \\ \mathrm{H} & 2.277681 & 2.834667 & 1.530601 \\ \mathrm{H} & 1.884907 & 2.364671 & -0.134087\end{array}$

\section{TS}

$\mathrm{H}$

C

C

$\mathrm{C}$

C

$\mathrm{N}$

C

$\mathrm{C}$

C

C

$\mathrm{N}$

O

$\mathrm{O}$

$\mathrm{H}$

C

$\mathrm{H}$

$\mathrm{H}$

$\mathrm{H}$

$\mathrm{H}$
(B3LYP/D95++(d,p))

0.000000

0.000000

0.961474

0.276515

$-1.090939$

$-1.239539$

$-2.140930$

$-1.764727$

0.743616

$-0.365660$

0.114886

1.911259

$-0.339809$

0.086037

$-3.570578$

$-2.511722$

1.097416

$-0.463508$

$-3.924692$
0.000000

0.000000

0.000000

$-0.191850$

$-0.319479$

$-0.201897$

$-0.569618$

$-0.617083$

$-0.358022$

$-0.256589$

$-1.102597$

$-0.558130$

0.952985

0.128289

$-0.716814$

$-0.716670$

$-1.366241$

$-1.890753$

0.198682
$E=-608.98056$ a.u.

0.000000

6.325790

5.336384

4.095106

4.372950

5.729928

3.390816

2.092244

2.732106

1.636958

0.436053

2.392962

0.977906

7.396623

3.850382

1.304867

0.582193

0.139132

4.345319 


$\begin{array}{cccc}\mathrm{H} & -4.233903 & -0.912646 & 3.003169 \\ \mathrm{H} & -3.681733 & -1.545089 & 4.564271 \\ \mathrm{H} & 2.026878 & 0.131763 & 5.471361 \\ \mathrm{H} & -2.118876 & -0.254249 & 6.225416\end{array}$

2TS

$\mathrm{H}$

C

C

C

C

C

C

C

C

$\mathrm{N}$

$\mathrm{O}$

$\mathrm{N}$

$\mathrm{O}$

$\mathrm{C}$

$\mathrm{H}$

$\mathrm{H}$

$\mathrm{H}$

$\mathrm{H}$

$\mathrm{H}$

$\mathrm{H}$

$\mathrm{H}$

$\mathrm{H}$

$\mathrm{H}$

\section{TS}

$\mathrm{H}$

C

$\mathrm{N}$

C

C

$\mathrm{N}$

C
(B3LYP/D95++(d,p))

0.000000

0.000000

$0.000000 \quad 0.000000$

0.746967

2.162617

2.809569

2.087354

0.697430

$-1.321433$

$-1.351237$

$-0.094767$

2.540385

$-0.101479$

0.790579

2.868081

3.859564

$-2.165294$

$-0.988800$

0.523747

3.924647

2.805469

2.414942

0.167891

$-2.154659$

(B3LYP/D95++(d,p))

0.000000

0.000000

$E=-625.03475$ a.u.

0.000000

0.000000

0.937381

0.000000

$\begin{array}{lll}0.252479 & -0.130849 \quad 4.068935\end{array}$

$\begin{array}{lll}-1.093066 & -0.224503 & 4.419917\end{array}$

$\begin{array}{lll}-1.236613 & -0.141466 & 5.783013\end{array}$

$\begin{array}{lll}-2.154241 & -0.423238 & 3.427049\end{array}$
$E=\mathbf{- 6 0 8 . 9 7 8 5 8}$ a.u.

0.000000

2.848018

4.022799

4.110827

1.716849

1.530299

3.177052

4.550623

5.053556

0.796805

0.520401

0.734109

5.437163

2.988596

5.195329

0.829263

0.101040

5.347156

5.819288

6.189118

6.025054
2.974318

2.501428 


\begin{tabular}{|c|c|c|c|}
\hline $\mathrm{C}$ & -1.781188 & -0.471873 & 2.127953 \\
\hline $\mathrm{C}$ & 0.746807 & -0.297043 & 2.726891 \\
\hline $\mathrm{C}$ & -0.370880 & -0.182971 & 1.646197 \\
\hline $\mathrm{N}$ & 0.054633 & -1.095248 & 0.466222 \\
\hline $\mathrm{O}$ & 1.922077 & -0.503512 & 2.414243 \\
\hline $\mathrm{O}$ & -0.283940 & 0.998820 & 0.949313 \\
\hline $\mathrm{H}$ & 0.260055 & 0.109765 & 7.296841 \\
\hline $\mathrm{C}$ & -3.579043 & -0.522586 & 3.902833 \\
\hline $\mathrm{H}$ & -2.533811 & -0.535128 & 1.342038 \\
\hline $\mathrm{H}$ & 1.017667 & -1.425171 & 0.594947 \\
\hline $\mathrm{H}$ & -0.581147 & -1.847697 & 0.194472 \\
\hline $\mathrm{H}$ & -3.875305 & 0.397095 & 4.422626 \\
\hline $\mathrm{H}$ & -4.262814 & -0.688931 & 3.064851 \\
\hline $\mathrm{H}$ & -3.692162 & -1.340009 & 4.625342 \\
\hline $\mathrm{H}$ & 1.941144 & 0.090430 & 5.359212 \\
\hline 4TS & \multicolumn{2}{|c|}{$($ B3LYP/D95++(d,p)) } & $E=-625.03232$ a.u. \\
\hline $\mathrm{H}$ & 0.000000 & 0.000000 & 0.000000 \\
\hline $\mathrm{C}$ & 0.000000 & 0.000000 & 2.843070 \\
\hline $\mathrm{C}$ & 0.605294 & 0.000000 & 4.084993 \\
\hline $\mathrm{C}$ & 2.056268 & 0.004597 & 4.241570 \\
\hline $\mathrm{C}$ & 2.831226 & 0.025372 & 3.115907 \\
\hline $\mathrm{C}$ & 2.270889 & 0.141845 & 1.768395 \\
\hline $\mathrm{C}$ & 0.722731 & -0.067598 & 1.535266 \\
\hline $\mathrm{N}$ & -1.342229 & -0.165949 & 3.090800 \\
\hline $\mathrm{C}$ & -1.491467 & -0.237504 & 4.457653 \\
\hline $\mathrm{N}$ & -0.338691 & -0.135770 & 5.089100 \\
\hline $\mathrm{O}$ & 2.954154 & 0.440452 & 0.787820 \\
\hline $\mathrm{N}$ & 0.308019 & 1.036628 & 0.507707 \\
\hline $\mathrm{O}$ & 0.374810 & -1.124302 & 0.744035 \\
\hline $\mathrm{C}$ & 2.628376 & -0.033292 & 5.628735 \\
\hline $\mathrm{H}$ & 3.916701 & 0.045973 & 3.179071 \\
\hline $\mathrm{H}$ & -2.460923 & -0.353258 & 4.926626 \\
\hline $\mathrm{H}$ & -0.352450 & 1.760772 & 0.797837 \\
\hline $\mathrm{H}$ & 1.152037 & 1.431675 & 0.076534 \\
\hline $\mathrm{H}$ & 3.721530 & -0.041775 & 5.611143 \\
\hline $\mathrm{H}$ & 2.266262 & -0.921951 & 6.160325 \\
\hline $\mathrm{H}$ & 2.280513 & 0.831361 & 6.207692 \\
\hline
\end{tabular}


5TS

$\mathrm{H}$

$\mathrm{C}$

$\mathrm{N}$

C

$\mathrm{C}$

$\mathrm{C}$

$\mathrm{C}$

$\mathrm{C}$

$\mathrm{C}$

$\mathrm{N}$

$\mathrm{O}$

$\mathrm{O}$

$\mathrm{H}$

$\mathrm{C}$

$\mathrm{H}$

$\mathrm{H}$

$\mathrm{H}$

$\mathrm{H}$

$\mathrm{H}$

$\mathrm{H}$

$\mathrm{C}$

$\mathrm{C}$

$\mathrm{H}$

$\mathrm{H}$

6TS

$\mathrm{H}$

$\mathrm{C}$

$\mathrm{C}$

$\mathrm{C}$

$\mathrm{C}$

$\mathrm{C}$

$\mathrm{C}$

$\mathrm{N}$

C
$(B 3 L Y P / D 95++(d, p)) \quad E=\mathbf{- 6 4 7 . 0 8 5 9 1}$ a.u.

$\begin{array}{lll}3.395162 & -0.356416 & 0.715376\end{array}$

$\begin{array}{lll}-2.765278 & -1.558989 & 0.147162\end{array}$

$\begin{array}{lll}-1.460470 & -1.827112 & 0.043902\end{array}$

$-0.614238 \quad-0.792823 \quad-0.047656$

$\begin{array}{lll}-1.011190 & 0.569890 & -0.053782\end{array}$

$\begin{array}{lll}-0.014067 & 1.663133 & -0.153640\end{array}$

$\begin{array}{lll}1.305410 & 1.384578 & -0.091336\end{array}$

$\begin{array}{lll}0.839382 & -1.109739 & -0.227862\end{array}$

$\begin{array}{lll}1.822342 & 0.000530 & 0.198970\end{array}$

$3.181418 \quad-0.302451 \quad-0.458103$

$1.247852 \quad-2.170058 \quad-0.678303$

$\begin{array}{lll}2.245294 & -0.167039 & 1.501370\end{array}$

$\begin{array}{lll}-3.430622 & -2.417528 & 0.237569\end{array}$

$\begin{array}{lll}-0.505249 & 3.087698 & -0.260449\end{array}$

$\begin{array}{lll}2.035813 & 2.193687 & -0.074147\end{array}$

$\begin{array}{lll}3.149452 & -1.220157 & -0.918912\end{array}$

$\begin{array}{lll}3.587786 & 0.418386 & -1.056949\end{array}$

$\begin{array}{lll}-1.079870 & 3.379497 & 0.628907\end{array}$

$\begin{array}{lll}0.337345 & 3.778664 & -0.353948\end{array}$

$\begin{array}{lll}-1.158025 & 3.226819 & -1.132228\end{array}$

$\begin{array}{lll}-2.393828 & 0.818116 & 0.027356\end{array}$

$\begin{array}{lll}-3.280486 & -0.253599 & 0.135751\end{array}$

$\begin{array}{lll}-2.772995 & 1.835849 & 0.013261\end{array}$

$\begin{array}{lll}-4.352643 & -0.087859 & 0.208849\end{array}$

$($ B3LYP/D95 $++(d, p)) \quad E=-647.10020$ a.u.

$2.081273 \quad-1.938702 \quad 0.919276$

$\begin{array}{lll}-0.316815 & -0.704396 & -0.015677\end{array}$

$\begin{array}{lll}-1.004211 & 0.532980 & 0.007450\end{array}$

$\begin{array}{lll}-0.244243 & 1.804685 & 0.041373\end{array}$

$\begin{array}{lll}1.107071 & 1.806658 & -0.108030\end{array}$

$\begin{array}{lll}1.863177 & 0.561281 & -0.310127\end{array}$

$\begin{array}{lll}1.180298 & -0.700235 & 0.236795\end{array}$

$\begin{array}{lll}-0.914137 & -1.889854 & -0.157288\end{array}$

$\begin{array}{lll}-2.255060 & -1.916784 & -0.234680\end{array}$ 


$\begin{array}{rrrr}\mathrm{O} & 2.984613 & 0.516517 & -0.811558 \\ \mathrm{~N} & 1.868033 & -1.948571 & -0.266550 \\ \mathrm{O} & 1.501236 & -0.851587 & 1.577326 \\ \mathrm{C} & -1.002890 & 3.099550 & 0.184525 \\ \mathrm{H} & 1.668777 & 2.736581 & -0.162720 \\ \mathrm{H} & -2.712788 & -2.897704 & -0.357604 \\ \mathrm{H} & 1.207899 & -2.646968 & -0.618908 \\ \mathrm{H} & 2.649406 & -1.741736 & -0.895906 \\ \mathrm{H} & -0.317000 & 3.949887 & 0.215950 \\ \mathrm{H} & -1.598182 & 3.102747 & 1.106960 \\ \mathrm{H} & -1.697408 & 3.252392 & -0.652119 \\ \mathrm{C} & -2.405368 & 0.477013 & -0.050944 \\ \mathrm{C} & -3.042426 & -0.763794 & -0.163731 \\ \mathrm{H} & -2.997596 & 1.387668 & -0.023158 \\ \mathrm{H} & -4.125639 & -0.836374 & -0.212774\end{array}$

7TS

$\mathrm{H}$

C

C

C

C

C

C

C

C

$\mathrm{N}$

$\mathrm{O}$

$\mathrm{O}$

$\mathrm{H}$

C

$\mathrm{H}$

$\mathrm{H}$

$\mathrm{H}$

$\mathrm{H}$

$\mathrm{H}$

$\mathrm{H}$

N
$(B 3 L Y P / D 95++(d, p)) \quad E=-647.09542$ a.u.

$\begin{array}{lll}0.000000 & 0.000000 & 0.000000\end{array}$

$\begin{array}{lll}0.000000 & 0.000000 & 6.452929\end{array}$

$\begin{array}{lll}0.616843 & 0.000000 & 5.201224\end{array}$

$\begin{array}{lll}-0.125451 & -0.402592 & 4.086205\end{array}$

$\begin{array}{lll}-1.469166 & -0.821921 & 4.261282\end{array}$

$-2.282672 \quad-1.277915 \quad 3.106326$

$\begin{array}{lll}-1.786311 & -1.174996 & 1.854686\end{array}$

$\begin{array}{lll}0.495885 & -0.480384 & 2.744602\end{array}$

$\begin{array}{lll}-0.476664 & -0.497089 & 1.548930\end{array}$

$\begin{array}{lll}0.265808 & -1.111014 & 0.343666\end{array}$

$\begin{array}{lll}1.710886 & -0.508572 & 2.568037\end{array}$

$\begin{array}{lll}-0.622139 & 0.761925 & 1.005468\end{array}$

$\begin{array}{lll}0.527259 & 0.316068 & 7.349022\end{array}$

$\begin{array}{lll}-3.673709 & -1.787082 & 3.383186\end{array}$

$\begin{array}{lll}-2.411751 & -1.423689 & 0.996910\end{array}$

$\begin{array}{lll}1.258914 & -1.243497 & 0.566854\end{array}$

$\begin{array}{lll}-0.146768 & -1.936752 & -0.094201\end{array}$

$\begin{array}{lll}-4.283234 & -1.007228 & 3.854232\end{array}$

$\begin{array}{lll}-4.159395 & -2.106575 & 2.455904\end{array}$

$\begin{array}{lll}-3.651994 & -2.627392 & 4.086553\end{array}$

$\begin{array}{lll}-2.051979 & -0.839274 & 5.475394\end{array}$ 


$\begin{array}{lrrc}\mathrm{C} & -1.333485 & -0.429473 & 6.528640 \\ \mathrm{H} & -1.847845 & -0.445942 & 7.490080 \\ \mathrm{H} & 1.651640 & 0.305678 & 5.066779\end{array}$

\begin{tabular}{lccc} 
8TS & \multicolumn{2}{c}{ (B3LYP/D95++(d,p)) } & $\mathbf{E}=\mathbf{- 6 4 7 . 0 9 5 7 1} \mathbf{a . u .}$ \\
$\mathrm{H}$ & 0.000000 & 0.000000 & 0.000000 \\
$\mathrm{C}$ & 0.000000 & 0.000000 & 2.840446 \\
$\mathrm{C}$ & 0.736217 & 0.000000 & 4.046198 \\
$\mathrm{C}$ & 2.183738 & 0.332844 & 4.054237 \\
$\mathrm{C}$ & 2.800203 & 0.764742 & 2.921807 \\
$\mathrm{C}$ & 2.058553 & 0.992288 & 1.683745 \\
$\mathrm{C}$ & 0.705711 & 0.256115 & 1.519066 \\
$\mathrm{C}$ & -1.340965 & -0.391367 & 2.889365 \\
$\mathrm{C}$ & -1.918521 & -0.707902 & 4.120679 \\
$\mathrm{O}$ & 2.471226 & 1.713500 & 0.774472 \\
$\mathrm{~N}$ & -0.127193 & 1.074784 & 0.508485 \\
$\mathrm{O}$ & 0.822460 & -0.866987 & 0.735115 \\
$\mathrm{C}$ & 2.927707 & 0.199356 & 5.353012 \\
$\mathrm{H}$ & 3.848263 & 1.056087 & 2.922924 \\
$\mathrm{H}$ & -2.961702 & -1.003565 & 4.197644 \\
$\mathrm{H}$ & -1.018190 & 1.473657 & 0.808875 \\
$\mathrm{H}$ & 0.487913 & 1.776946 & 0.076848 \\
$\mathrm{H}$ & 3.983111 & 0.455579 & 5.225832 \\
$\mathrm{H}$ & 2.844496 & -0.824262 & 5.736453 \\
$\mathrm{H}$ & 2.484626 & 0.845594 & 6.119382 \\
$\mathrm{~N}$ & 0.185224 & -0.327358 & 5.232979 \\
$\mathrm{C}$ & -1.108983 & -0.656135 & 5.264488 \\
$\mathrm{H}$ & -1.520598 & -0.901997 & 6.243419 \\
$\mathrm{H}$ & -1.923845 & -0.472746 & 1.973615 \\
& & &
\end{tabular}

\begin{tabular}{|c|c|c|c|}
\hline 9TS & \multicolumn{2}{|c|}{$($ B3LYP/D95++(d,p)) } & $E=-663.12813$ a.u. \\
\hline $\mathrm{H}$ & 0.000000 & 0.000000 & 0.000000 \\
\hline $\mathrm{C}$ & 0.000000 & 0.000000 & 6.276788 \\
\hline $\mathrm{N}$ & 0.597412 & 0.000000 & 5.080435 \\
\hline $\mathrm{C}$ & -0.122865 & -0.480852 & 4.061678 \\
\hline $\mathrm{C}$ & -1.439161 & -0.974296 & 4.209902 \\
\hline $\mathrm{C}$ & -2.216687 & -1.486963 & 3.059266 \\
\hline $\mathrm{C}$ & -1.723219 & -1.357572 & 1.808969 \\
\hline
\end{tabular}




$\begin{array}{lrrr}\mathrm{C} & 0.524065 & -0.578683 & 2.707774 \\ \mathrm{C} & -0.453614 & -0.598938 & 1.516586 \\ \mathrm{~N} & 0.311346 & -1.118690 & 0.288026 \\ \mathrm{O} & 1.732036 & -0.640807 & 2.547563 \\ \mathrm{O} & -0.669805 & 0.674837 & 1.033105 \\ \mathrm{H} & 0.572010 & 0.408198 & 7.109171 \\ \mathrm{C} & -3.579008 & -2.087878 & 3.312206 \\ \mathrm{H} & -2.326615 & -1.651172 & 0.949959 \\ \mathrm{H} & 1.307582 & -1.234624 & 0.508950 \\ \mathrm{H} & -0.073080 & -1.932102 & -0.195811 \\ \mathrm{H} & -4.263701 & -1.350143 & 3.751402 \\ \mathrm{H} & -4.023096 & -2.441423 & 2.377438 \\ \mathrm{H} & -3.524093 & -2.936407 & 4.006581 \\ \mathrm{C} & -1.931918 & -0.944068 & 5.528831 \\ \mathrm{~N} & -1.229525 & -0.458740 & 6.559166 \\ \mathrm{H} & -2.926796 & -1.318805 & 5.762141\end{array}$

\begin{tabular}{lrcc} 
10TS & \multicolumn{2}{c}{ (B3LYP/D95++(d,p) $)$} & $\mathbf{E}=\mathbf{- 6 6 3 . 1 4 4 1 9} \mathbf{a . u .}$ \\
$\mathrm{H}$ & 0.000000 & 0.000000 & 0.000000 \\
$\mathrm{C}$ & 0.000000 & 0.000000 & 2.845281 \\
$\mathrm{C}$ & 0.875121 & 0.000000 & 3.953520 \\
$\mathrm{C}$ & 2.186897 & 0.679878 & 3.878920 \\
$\mathrm{C}$ & 2.499824 & 1.439818 & 2.795724 \\
$\mathrm{C}$ & 1.561527 & 1.618651 & 1.676144 \\
$\mathrm{C}$ & 0.521748 & 0.498192 & 1.512538 \\
$\mathrm{~N}$ & -1.234795 & -0.504041 & 2.900628 \\
$\mathrm{C}$ & -1.599001 & -1.065022 & 4.065277 \\
$\mathrm{O}$ & 1.623457 & 2.547901 & 0.875021 \\
$\mathrm{~N}$ & -0.572678 & 0.907083 & 0.555144 \\
$\mathrm{O}$ & 1.052894 & -0.527240 & 0.747594 \\
$\mathrm{C}$ & 3.133619 & 0.561413 & 5.045307 \\
$\mathrm{H}$ & 3.425830 & 2.008850 & 2.753393 \\
$\mathrm{H}$ & -2.609553 & -1.468722 & 4.116761 \\
$\mathrm{H}$ & -1.514749 & 0.723886 & 0.910079 \\
$\mathrm{H}$ & -0.435769 & 1.853026 & 0.185521 \\
$\mathrm{H}$ & 4.063540 & 1.100294 & 4.847111 \\
$\mathrm{H}$ & 3.379361 & -0.490245 & 5.242540 \\
$\mathrm{H}$ & 2.690485 & 0.969005 & 5.963451
\end{tabular}




$\begin{array}{lrrr}\mathrm{C} & 0.380919 & -0.639233 & 5.099795 \\ \mathrm{~N} & -0.847600 & -1.178721 & 5.164406 \\ \mathrm{H} & 0.981531 & -0.716200 & 6.004514\end{array}$

11TS

$\mathrm{H}$

C

$\mathrm{N}$

C

C

C

C

C

C

$\mathrm{N}$

O

$\mathrm{O}$

$\mathrm{H}$

C

$\mathrm{N}$

$\mathrm{H}$

$\mathrm{H}$

C

$\mathrm{H}$

$\mathrm{H}$

C

C

$\mathrm{H}$

$\mathrm{H}$

$\mathrm{H}$

12TS

$\mathrm{H}$

$\mathrm{C}$

C

C

C

C
(B3LYP/D95++(d,p)) $\quad E=-\mathbf{7 3 9 . 3 8 2 8 8}$ a.u.

$\begin{array}{lll}-0.875504 & 2.900345 & 0.844602\end{array}$

$3.079888 \quad-1.741671 \quad 0.042749$

$2.993606 \quad-0.385073 \quad-0.114824$

$\begin{array}{lll}1.670842 & -0.008223 & -0.078861\end{array}$

$\begin{array}{lll}0.890806 & -1.159544 & 0.084910\end{array}$

$\begin{array}{lll}-0.571840 & -1.081637 & 0.043519\end{array}$

$\begin{array}{lll}-1.170724 & 0.201350 & -0.015336\end{array}$

$\begin{array}{lll}1.136787 & 1.326122 & -0.269169\end{array}$

$\begin{array}{lll}-0.314636 & 1.444164 & 0.233596\end{array}$

$\begin{array}{lll}-0.962493 & 2.696951 & -0.329776\end{array}$

$\begin{array}{lll}1.771020 & 2.294612 & -0.696398\end{array}$

$\begin{array}{lll}-0.299805 & 1.832505 & 1.557240\end{array}$

$\begin{array}{lll}4.035972 & -2.248053 & 0.066828\end{array}$

$\begin{array}{lll}-1.424793 & -2.192062 & -0.011652\end{array}$

$\begin{array}{lll}-2.481222 & 0.399059 & -0.187090\end{array}$

$\begin{array}{lll}-0.341789 & 3.221874 & -0.951461\end{array}$

$\begin{array}{lll}-1.898570 & 2.511717 & -0.701459\end{array}$

$\begin{array}{lll}1.790759 & -2.259692 & 0.160328\end{array}$

$\begin{array}{lll}1.549465 & -3.307501 & 0.284423\end{array}$

$\begin{array}{lll}3.760276 & 0.267441 & -0.212349\end{array}$

$\begin{array}{lll}-2.803733 & -1.988928 & -0.146051\end{array}$

$\begin{array}{lll}-3.282017 & -0.677895 & -0.251010\end{array}$

$\begin{array}{lll}-1.018452 & -3.200343 & 0.032322\end{array}$

$\begin{array}{lll}-4.344376 & -0.483412 & -0.394918\end{array}$

$\begin{array}{lll}-3.494016 & -2.827456 & -0.191029\end{array}$

(B3LYP/D95++(d,p)) $\quad E=-739.36127$ a.u.

$\begin{array}{lll}-2.749869 & -2.000197 & 0.223780\end{array}$

$\begin{array}{lll}-1.401520 & 0.480594 & -0.023834\end{array}$

$\begin{array}{lll}-0.293224 & 1.309311 & -0.126358\end{array}$

$\begin{array}{lll}1.061874 & 0.767922 & -0.076355\end{array}$

$\begin{array}{lll}1.255374 & -0.634124 & 0.039465\end{array}$

$\begin{array}{lll}0.073276 & -1.562284 & -0.043885\end{array}$ 


$\begin{array}{lrrr}\mathrm{C} & -1.377140 & -1.001375 & 0.179577 \\ \mathrm{~N} & -2.521897 & 1.271018 & 0.056693 \\ \mathrm{C} & -2.152027 & 2.600402 & -0.004991 \\ \mathrm{O} & 0.197290 & -2.745996 & -0.323148 \\ \mathrm{~N} & -2.278212 & -1.788654 & -0.846132 \\ \mathrm{O} & -2.019833 & -1.455437 & 1.290627 \\ \mathrm{~N} & 2.462275 & -1.215078 & 0.142249 \\ \mathrm{H} & -2.888181 & 3.391700 & 0.038995 \\ \mathrm{H} & -2.736254 & -1.273131 & -1.600084 \\ \mathrm{H} & -1.756302 & -2.601504 & -1.192095 \\ \mathrm{C} & -0.775071 & 2.661256 & -0.129563 \\ \mathrm{H} & -0.193525 & 3.571170 & -0.203993 \\ \mathrm{C} & 2.216065 & 1.573679 & -0.099965 \\ \mathrm{C} & 3.468120 & 0.971693 & 0.007587 \\ \mathrm{H} & 2.126822 & 2.653584 & -0.192297 \\ \mathrm{C} & 3.539364 & -0.428222 & 0.136923 \\ \mathrm{H} & 4.504304 & -0.925520 & 0.235717 \\ \mathrm{H} & -3.431957 & 0.926225 & 0.331573 \\ \mathrm{H} & 4.379601 & 1.565025 & -0.002696\end{array}$

\section{TS}

$\mathrm{H}$

$\mathrm{C}$

$\mathrm{N}$

$\mathrm{C}$

$\mathrm{C}$

$\mathrm{C}$

$\mathrm{C}$

$\mathrm{C}$

$\mathrm{C}$

$\mathrm{N}$

$\mathrm{O}$

$\mathrm{O}$

$\mathrm{H}$

$\mathrm{C}$

$\mathrm{N}$

$\mathrm{H}$

$\mathrm{H}$

$($ B3LYP/D95++(d,p)) $\quad E=\mathbf{- 7 5 5 . 4 2 6 8 5}$ a.u.

$\begin{array}{lll}-0.964525 & 2.878189 & 0.833932\end{array}$

$3.105968 \quad-1.684611 \quad 0.044310$

$\begin{array}{lll}2.992765 & -0.329807 & -0.108971\end{array}$

$\begin{array}{lll}1.663005 & 0.020951 & -0.075457\end{array}$

$0.907303 \quad-1.148195 \quad 0.083593$

$\begin{array}{lll}-0.554053 & -1.089337 & 0.040230\end{array}$

$\begin{array}{lll}-1.177302 & 0.178247 & -0.017755\end{array}$

$\begin{array}{lll}1.105483 & 1.346223 & -0.260165\end{array}$

$\begin{array}{lll}-0.354642 & 1.440695 & 0.228043\end{array}$

$\begin{array}{lll}-1.021161 & 2.677439 & -0.345578\end{array}$

$\begin{array}{lll}1.723343 & 2.329894 & -0.674796\end{array}$

$\begin{array}{lll}-0.374715 & 1.824282 & 1.551514\end{array}$

$\begin{array}{lll}4.072011 & -2.171674 & 0.067949\end{array}$

$-1.418983-2.189805 \quad-0.017200$

$\begin{array}{lll}-2.495089 & 0.318554 & -0.189289\end{array}$

$\begin{array}{lll}-0.396222 & 3.220216 & -0.948034\end{array}$

$\begin{array}{lll}-1.944334 & 2.484477 & -0.743347\end{array}$ 


$\begin{array}{lrrc}\mathrm{C} & 1.827625 & -2.230291 & 0.157670 \\ \mathrm{H} & 1.606442 & -3.282933 & 0.277085 \\ \mathrm{H} & 3.747164 & 0.337607 & -0.203322 \\ \mathrm{~N} & -2.749774 & -2.062587 & -0.149346 \\ \mathrm{C} & -3.216605 & -0.813188 & -0.247690 \\ \mathrm{H} & -1.032913 & -3.207869 & 0.031942 \\ \mathrm{H} & -4.290617 & -0.700755 & -0.391129\end{array}$

14TS

$\mathrm{H}$

C

$\mathrm{C}$

C

C

C

C

$\mathrm{N}$

C

$\mathrm{O}$

$\mathrm{N}$

$\mathrm{O}$

$\mathrm{N}$

$\mathrm{H}$

$\mathrm{H}$

$\mathrm{H}$

C

$\mathrm{H}$

C

$\mathrm{N}$

$\mathrm{H}$

C

$\mathrm{H}$

$\mathrm{H}$

15TS

$\mathrm{H}$

C

C
$($ B3LYP/D95++(d,p)) $\quad E=\mathbf{- 7 5 5 . 4 0 3 6 8}$ a.u.

$\begin{array}{lll}-2.737318 & -2.008549 & 0.237072\end{array}$

$\begin{array}{lll}-1.392422 & 0.477280 & -0.023752\end{array}$

$\begin{array}{lll}-0.285859 & 1.309908 & -0.129452\end{array}$

$\begin{array}{lll}1.064125 & 0.763082 & -0.078002\end{array}$

$\begin{array}{lll}1.261849 & -0.631892 & 0.042276\end{array}$

$\begin{array}{lll}0.080167 & -1.568094 & -0.047202\end{array}$

$\begin{array}{lll}-1.368312 & -1.006476 & 0.179623\end{array}$

$\begin{array}{lll}-2.512721 & 1.266837 & 0.057803\end{array}$

$\begin{array}{lll}-2.145083 & 2.596820 & -0.006796\end{array}$

$\begin{array}{lll}0.217471 & -2.746142 & -0.334558\end{array}$

$\begin{array}{lll}-2.276426 & -1.792924 & -0.838821\end{array}$

$\begin{array}{lll}-1.999875 & -1.461399 & 1.295798\end{array}$

$\begin{array}{lll}2.480219 & -1.182303 & 0.149565\end{array}$

$\begin{array}{lll}-2.882659 & 3.386675 & 0.038016\end{array}$

$\begin{array}{lll}-2.745478 & -1.273861 & -1.583666\end{array}$

$\begin{array}{lll}-1.763301 & -2.604839 & -1.198774\end{array}$

$\begin{array}{lll}-0.768640 & 2.661043 \quad-0.133460\end{array}$

$\begin{array}{lll}-0.187613 & 3.571031 & -0.208778\end{array}$

$\begin{array}{lll}2.243445 & 1.535781 & -0.100221\end{array}$

$\begin{array}{lll}3.461528 & 0.999423 & 0.005372\end{array}$

$2.194530 \quad 2.619971 \quad-0.199945$

$\begin{array}{lll}3.515636 & -0.339469 & 0.136980\end{array}$

$\begin{array}{lll}4.507373 & -0.778039 & 0.238565\end{array}$

$\begin{array}{lll}-3.423946 & 0.922816 & 0.330192\end{array}$

(B3LYP/D95++(d,p)) $\quad E=-\mathbf{7 3 9 . 3 7 8 2 5}$ a.u.

$\begin{array}{lll}-0.957984 & 2.867408 & 0.901436\end{array}$

$\begin{array}{lll}3.107233 & -1.691305 & 0.059325\end{array}$

$\begin{array}{lll}3.100122 & -0.321189 & -0.122758\end{array}$ 


$\begin{array}{lrrr}\mathrm{C} & 1.732555 & 0.090093 & -0.102483 \\ \mathrm{C} & 0.942468 & -1.052198 & 0.076500 \\ \mathrm{C} & -0.513566 & -1.071675 & 0.032196 \\ \mathrm{C} & -1.164858 & 0.187837 & -0.015316 \\ \mathrm{C} & 1.098521 & 1.393554 & -0.292421 \\ \mathrm{C} & -0.347931 & 1.451518 & 0.247235 \\ \mathrm{~N} & -1.043941 & 2.693041 & -0.277875 \\ \mathrm{O} & 1.640717 & 2.392587 & -0.763357 \\ \mathrm{O} & -0.336572 & 1.803664 & 1.581375 \\ \mathrm{H} & 3.930372 & -2.389596 & 0.129560 \\ \mathrm{C} & -1.316476 & -2.219162 & -0.035635 \\ \mathrm{~N} & -2.481703 & 0.328317 & -0.182388 \\ \mathrm{H} & -0.432999 & 3.248379 & -0.884193 \\ \mathrm{H} & -1.973053 & 2.494092 & -0.657848 \\ \mathrm{~N} & 1.793314 & -2.123791 & 0.168634 \\ \mathrm{C} & -2.703147 & -2.072533 & -0.165502 \\ \mathrm{C} & -3.236320 & -0.781835 & -0.254994 \\ \mathrm{H} & -0.879082 & -3.216010 & -0.007041 \\ \mathrm{H} & -4.306380 & -0.631752 & -0.393847 \\ \mathrm{H} & -3.356583 & -2.939401 & -0.219189 \\ \mathrm{H} & 1.513417 & -3.084087 & 0.309134 \\ \mathrm{H} & 3.963841 & 0.319369 & -0.237653\end{array}$

16TS

$\mathrm{H}$

$\mathrm{C}$

$\mathrm{C}$

$\mathrm{C}$

C

C

$\mathrm{C}$

C

$\mathrm{C}$

$\mathrm{O}$

$\mathrm{N}$

$\mathrm{O}$

$\mathrm{N}$

$\mathrm{H}$
(B3LYP/D95++(d,p)) $\quad E=-\mathbf{7 3 9 . 3 6 1 3 9}$ a.u.

$\begin{array}{lll}-2.572844 & -2.078395 & 0.734650\end{array}$

$\begin{array}{lll}-1.483835 & 0.459658 & -0.016608\end{array}$

$\begin{array}{lll}-0.342205 & 1.248444 & -0.107889\end{array}$

$\begin{array}{lll}1.020872 & 0.751081 & -0.033056\end{array}$

$\begin{array}{lll}1.218656 & -0.656934 & -0.058086\end{array}$

$\begin{array}{lll}0.019215 & -1.548022 & -0.252953\end{array}$

$\begin{array}{lll}-1.350691 & -1.016905 & 0.249785\end{array}$

$\begin{array}{lll}-2.611275 & 1.329288 & -0.038846\end{array}$

$\begin{array}{lll}-2.124285 & 2.624441 & -0.161700\end{array}$

$\begin{array}{lll}0.105136 & -2.655347 & -0.766620\end{array}$

$\begin{array}{lll}-2.441859 & -1.876628 & -0.427365\end{array}$

$\begin{array}{lll}-1.598071 & -1.433453 & 1.531240\end{array}$

$\begin{array}{lll}2.420894 & -1.241526 & 0.007674\end{array}$

$\begin{array}{lll}-2.648909 & 3.568081 & -0.229992\end{array}$ 


$\begin{array}{lrrc}\mathrm{H} & -3.138040 & -1.394358 & -0.997382 \\ \mathrm{H} & -1.992991 & -2.654312 & -0.927221 \\ \mathrm{~N} & -0.749063 & 2.567045 & -0.190707 \\ \mathrm{H} & -0.140162 & 3.364652 & -0.301182 \\ \mathrm{C} & 2.169089 & 1.559174 & 0.059714 \\ \mathrm{C} & 3.421568 & 0.949223 & 0.136289 \\ \mathrm{H} & 2.091730 & 2.644804 & 0.085584 \\ \mathrm{C} & 3.496429 & -0.454215 & 0.113643 \\ \mathrm{H} & 4.460817 & -0.957232 & 0.180814 \\ \mathrm{H} & -3.656399 & 1.060763 & 0.052565\end{array}$




\section{Appendix 2}

(Natural population analysis at the B3LYP/D95(d,p) for OBQ, MIQ and IIQ)

\begin{tabular}{|c|c|c|}
\hline OBQ & MIQ & IIQ \\
\hline C $\quad 0.47775$ & C $\quad 0.49445$ & C $\quad 0.48597$ \\
\hline C -0.26305 & -0.31578 & -0.28811 \\
\hline C -0.17949 & 0.46915 & 0.46848 \\
\hline C -0.17949 & -0.52332 & -0.52268 \\
\hline C $\quad-0.26305$ & 0.06344 & -0.01088 \\
\hline C $\quad 0.47775$ & 0.08615 & 0.08610 \\
\hline $\mathrm{O} \quad-0.49668$ & 0.22563 & 0.23340 \\
\hline H $\quad 0.23386$ & -0.54984 & -0.55072 \\
\hline H $\quad 0.22761$ & -0.10677 & -0.10137 \\
\hline H $\quad 0.22761$ & -0.53944 & 0.16739 \\
\hline H $\quad 0.23386$ & -0.64174 & -0.53756 \\
\hline $\mathrm{O} \quad-0.49668$ & -0.05806 & -0.05728 \\
\hline & 0.02064 & 0.02119 \\
\hline & 0.44525 & 0.44591 \\
\hline & 0.22871 & -0.03730 \\
\hline & 0.22871 & -0.60273 \\
\hline & 0.22874 & -0.64515 \\
\hline & 0.21357 & 0.21430 \\
\hline & -0.64515 & -0.64803 \\
\hline & 0.22558 & 0.42652 \\
\hline & 0.22503 & -0.08830 \\
\hline & 0.22503 & 0.20048 \\
\hline & & -0.25108 \\
\hline & & -0.20057 \\
\hline & & 0.22196 \\
\hline & & 0.22449 \\
\hline & & 0.23685 \\
\hline & & 0.22994 \\
\hline & & 0.22453 \\
\hline & & 0.22815 \\
\hline & & 0.21482 \\
\hline & & -0.20800 \\
\hline & & -0.23035 \\
\hline & & 0.21631 \\
\hline & & 0.21682 \\
\hline & & 0.21651 \\
\hline
\end{tabular}

(END) 